PART I: STI PRODUCT DESCRIPTION

(To be completed by Recipient/Contractor)

A. STI Product Identifiers

1. REPORT/PRODUCT NUMBER(s)

None

2. DOE AWARD/CONTRACT NUMBER(s)

DE-FC36-97ID13554

3. OTHER IDENTIFYING NUMBER(s)

None

\section{B. Recipient/Contractor}

Carnegie Mellon University, Dept. of Materials Science and

Engineering, 5000 Forbes Ave., Pittsburgh, PA 15213

C. STI Product Title

Hydrogen and Nitrogen Control in Ladle and Casting Operations

D. Author(s)

Richard J. Fruehan

Siddhartha Misra

E-mail Address(es):

Fruehan@andrew.cmu.edu

E STI Product Issue Date/Date of Publication

$\underline{01} \underline{15} \underline{2005}$

$M M$ DD YYYY

F. STI Product Type (Select only one)

风 1. TECHNICAL REPORT

囚 Final $\square$ Other (specify)

$\square$ 2. CONFERENCE PAPER/PROCEEDINGS

Conference Information (title, location, dates)

$\square \quad$ 3. JOURNAL ARTICLE
a. TYPE: $\square$ Announcement Citation Only
$\square$ Preprint $\square$ Postprint
b. JOURNAL NAME

c. VOLUME d. ISSUE

e. SERIAL IDENTIFIER (e.g. ISSN or CODEN)

$\square$ 4. OTHER, SPECIFY

G. STI Product Reporting Period
$\underline{03} \quad \underline{01} \quad \underline{2001}$
$\overline{M M} \quad \overline{D D} \quad \overline{Y Y Y Y}$
Thru $-\frac{01}{M M} \frac{13}{D D} \quad \frac{2005}{Y Y Y Y}$

H. Sponsoring DOE Program Office

Office of Industrial Technologies (OIT)(EE20)

I. Subject Categories (list primary one first)

32 Energy Conservation, Consumption and Utilization Keywords: Steel

\section{J. Description/Abstract}

The ultimate goal of the project was to develop a set of equations which could predict the nitrogen and hydrogen pickup from various sources during ladle and casting operations. The comprehensive model is designed to predict the gas pickup under varying operating conditions such as the metal oxygen and sulfur content, the total tapping or stirring time, the stirring gas flow rate and the slag thickness. The model predictions are based on mathematical and empirical evidence which are derived from thermodynamic and kinetic fundamental principles.

K. Intellectual Property/Distribution Limitations

(must select at least one; if uncertain contact your

Contracting Officer (CO))

囚 1. UNLIMITED ANNOUNCEMENT (available to U.S. and non-U.S. public; the Government assumes no liability for disclosure of such data)

2. COPYRIGHTED MATERIAL: Are there any restrictions based on copyright? $\square$ Yes $\square$ No.

If yes, list the restrictions as contained in your award document

$\square$ 3. PATENTABLE MATERIAL: THERE IS PATENTABLE MATERIAL IN THE DOCUMENT. INENTION DISCLOSURE SUBMITTED TO DOE: DOE Docket Number: S-

(Sections are marked as restricted distribution pursuant to 35 USC 205)

$\square$ 4. PROTECTED DATA: $\square$ CRADA $\square$ Other, specify

Release date (required) no more than

5 years from date listed in Part I.E. above $\overline{\mathrm{MM}} \overline{\mathrm{DD}} \overline{\mathrm{YYYY}}$

5. SMALL BUSINESS INNOVATION RESEARCH (SBIR) DATA

Release date (required) no more than 4

years from date listed in Part I.E. above $\overline{\mathrm{MM}} \overline{\mathrm{DD}} \overline{\mathrm{YYYY}}$

6. SMALL BUSINESS TECHNOLOGY TRANSFER RESEARCH (STTR) DATA

Release date (required) no more than 4

years from date listed in Part I.E. above $\overline{\mathrm{MM}} \overline{\mathrm{DD}} \overline{\mathrm{YYYY}}$

7. OFFICE OF NUCLEAR ENERGY APPLICED TECHNOLOGY

L. Recipient/Contract Point of Contact Contact for additional information (contact or organization name To be included in published citations and who would Receive any external questions about the content of the STI Product or the research contained herein)

Dr. Richard Fruehan

Name and/or Position

fruehan@andrew.cmu.edu

E-mail (412) 268-2677

Dept. of Mat'I Science and Engineering, Carnegie Mellon University, Pittsburgh, PA 15213 


\section{PART II: STI PRODUCT MEDIA/FORMAT and} LOCATION/TRANSMISSION

(To be completed by Recipient/Contractor)

A. Media/Format Information:

1. MEDIUM OF STI PRODUCT IS:

X Electronic Document $\quad \square$ Computer Medium

$\square$ Audiovisual Material $\square$ Paper $\square$ No Fulltext

2. SIZE OF STI PRODUCT 62 Pages

3. SPECIFY FILE FORMAT OF ELECTRONIC DOCUMENT

BEING TRANSMITTED, INDICATE:

$\square$ SGML $\square$ HTML $\square$ XML ХXPDF Normal

$\square$ PDF Image $\square$ TIFFG4

$\square$ WP-indicate Version (5.0 or greater)

Platform/operation system

$\square$ MS-indicate Version (5.0 or greater)

Platform/operation system

$\square$ Postscript

4. IF COMPUTER MEDIUM OR AUDIOVISUAL MATERIAL:

a. Quantity/type (specify)

b. Machine compatibility (specify)

c. Other information about product format a user needs to know:

B. Transmission Information:

1. STI PRODUCT IS BEING TRANSMITTED:

X a. Electronically via E-Link

b. Via mail or shipment to address indicated in award document (Paper product, CD-ROM, diskettes, video cassettes, etc.)

2. INFORMATION PRODUCT FILE NAME

囚 (of transmitted electronic format) EditedTRP0006FinalReport.

\section{PART III: STI PRODUCT REVIEW/RELEASE INFORMATION \\ (To be completed by DOE)}

A. STI Product Reporting Requirements Review.

1. THIS DELIVERABLE COMPLETES ALL REQUIRED DELIVERABLES FOR THIS AWARD

$\square$ 2. THIS DELIVERABLE FULFILLS A TECHNICAL INFORMATION REPORTING REQUIREMENT, BUT SHOULD NOT BE DISSEMINATED BEYOND DOE.

B. Award Office Is the Source of STI Product Availability THE STI PRODUCT IS NOT AVAILABLE IN AN ELECTRONIC MEDIUM. THE AWARDING OFFICE WILL SERVE AS THE INTERIM SOURCE OF AVAILABILITY.

C. DOE Releasing Official

$\square$ 1. I VERIFY THAT ALL NECESSARY REVIEWS HAVE BEEN COMPLETED AS DESCRIBED IN DOE G 241.1-1A, PART II, SECTION 3.0 AND THAT THE STI PRODUCT SHOULD BE RELEASED IN ACCORDANCE WITH THE INTELLECTUAL PROPERTY/DISTRIBUTION LIMITATION ABOVE.

Release by (name)

Date $\overline{\mathrm{MM}} \overline{\mathrm{DD}} \overline{\mathrm{YYYY}}$

E-Mail

Phone 


\title{
Hydrogen and Nitrogen Control in Ladle and Casting Operations
}

\author{
Prepared
}

By

\author{
R. J. Fruehan \\ \& \\ Siddhartha Misra
}
Materials Science and Engineering Department
Carnegie Mellon University
Pittsburgh, PA

January $15^{\text {th }}, 2005$

Industrial Partners:

US Steel, Arcelor, Corus, Iscor Ltd., CVRD,

AK Steel, Ispat Inland Steel Company, Timken Company

International Steel Group, Siderar, S. A. I. C.

Bao Steel, Gerdau Ameristeel

Work Performed Under Agreement DE-FC36-97ID 13554 


\title{
Report Documentation Page
}

Title and Subtitle:

Author(s):

Performing Organization
AISI/DOE Technology Roadmap Program

Hydrogen and Nitrogen Control in Ladle and Casting

Operations

TRP 0006

Richard J. Fruehan, Principal Investigator

Siddhartha Misra

Carnegie Mellon University

Pittsburgh, PA

\begin{abstract}
:
In recent years there has been an increasing demand to reduce and control the amount of dissolved gases in steel. Hydrogen and nitrogen are two of the most important gases which when dissolved in liquid steel affect its properties significantly. Several steelmaking additions have been investigated in this research for their effect on the hydrogen and nitrogen content of steels. It has been established that calcium hydroxide (hydrated lime) acts as a source of hydrogen. Carburizers, such as metallurgical coke, were found to result in no hydrogen pickup when added to liquid steel. Addition of petroleum coke, on the other hand, increased the hydrogen content of liquid steel. Ferroalloy such as medium carbon ferromanganese when added to the liquid iron was found to increase its nitrogen content, the increase being proportional to the amount of ferroalloy added. Similarly, addition of pitch coke, which had a significant nitrogen impurity, increased the nitrogen content of liquid iron. A mathematical model was developed to quantify the absorption of nitrogen and hydrogen from the air bubbles entrained during tapping of liquid steel. During the bottom stirring of liquid metal in a ladle, the inert gas escaping from the top displaces the slag layer and often forms an open eye. The absorption of atmospheric nitrogen through the spout eye was estimated for different slag thickness and gas flow rate.

The ultimate goal of this research was to develop a comprehensive set of equations which could predict the nitrogen and hydrogen pickup from their various sources. Estimates of hydrogen and nitrogen pickup during the steel transfer operations such as tapping and ladle stirring and the predicted pickup from steelmaking additions were integrated into empirical equations. The comprehensive model is designed to predict the gas pickup under varying operating conditions such as the metal oxygen and sulfur content, the total tapping or stirring time, the stirring gas flow rate and the slag thickness. The model predictions are based on mathematical and empirical evidence which are derived from thermodynamic and kinetic fundamental principles.
\end{abstract}




\section{DISCLAIMER}

"This report was prepared as an account of work sponsored by an Agency of the United States Government. Neither the United States Government nor any agency thereof, nor any of their employees, makes any warranty, express or implied, or assumes any legal liability or responsibility for the accuracy, completeness, or usefulness of any information, apparatus, product, or process disclosed, or represents that it use would not infringe privately owned rights. Reference herein to any specific commercial product, process, or service by trade name, trademark, manufacturer, or otherwise, does not necessarily constitute or imply endorsement, recommendation, or favoring by the United States Government or any agency thereof. The views and opinions of authors expressed herein do not necessarily state or reflect those of the United States Government or any agency thereof."

"This report has been reproduced from the best available copy. Available in paper copy and microfiche"

Number of pages in report: 62

DOE and DOE contractors can obtain copies of this report from:

Office of Scientific and Technical Information,

P.O. Box 62, Oak Ridge, TN 37831.

(865) 576-8401

This report is publicly available from the Department of Commerce,

National Technical Information Service, 5285 Port Royal Road,

Springfield, VA 22161.

(703) 605-6000 (1-800-553-6847). 


\section{TABLE OF CONTENTS}

ITEM

PAGE

Report Documentation Information

Disclaimer

ii

Table of Contents

iii

List of Figures

iv

List of Tables

v

Executive Summary

2

Introduction

3

Objective

4

Research Results

4

Appendix A: Research Details by Task

Task I - Hydrogen Pickup from $\mathrm{CA}(\mathrm{OH})_{2}$

Task II - Hydrogen and Nitrogen Pickup from Alloys and

Carburizers

12

Task III - Transport of Nitrogen through Slag

Task IV - Model of Ladle Processing 


\section{LIST OF FIGURES}

FIGURE

TITLE

PAGE

1 Hydrogen pickup due to $\mathrm{CA}(\mathrm{OH})_{2}$ addition on top of the slag

2 Hydrogen pickup due to $\mathrm{CA}(\mathrm{OH}) 2$ addition along with the slag

3

Change in the hydrogen content of the liqiud metal (at the LRS) as a function of coke addition for 90 tonne heat

$4 \quad$ Nitrogen pickup from medium carbon ferromanganese, Trial 1

$5 \quad$ Nitrogen pickup from medium carbon ferromanganese, Trial 2

6 Effect of pitch coke addition on the nitrogen and carbon content of iron, Trial 1

7 Effect of pitch coke addition on the nitrogen and carbon content of iron, Trial 2

8 Effect of pitch coke addition on the nitrogen and carbon content of iron, Trial 3

9 Effect of pitch coke addition on the nitrogen and carbon content of iron, Trial 4

10 Nitrogen pickup corrected for the desorption, Trial 3

11 Nitrogen pickup corrected for the desorption, Trial 4

Effect of pitch coke (heated to $1823 \mathrm{~K}$ ) addition on the nitrogen and carbon content of iron, Trial 5

13 Nitrogen pickup through a slag layer as a function of time, Trials 1 and 2

14 Schematic representation of the nitrogen diffusion through the slag layer

15 Nitrogen transport through $\mathrm{MgO}$ rich slag, Trial 3

16 Nitrogen transport through $\mathrm{Al}_{2} \mathrm{O}_{3}$ rich slag, Trial 4

17 Nitrogen transport through slag layer, calculated and experimental, Trial 2

18 Nitrogen transport through slag layer, calculated and experimental, Trial 3

19 Nitrogen transport through slag layer, calculated and experimental, Trial 4

20 Water vapor partial pressure as a function of temperature 21 Nitrogen pickup for different oxygen activity at the bubble surface, $\mathrm{N}_{\mathrm{i}}=30 \mathrm{ppm}$,
$\% \mathrm{~S}=0.005 \%$

Hydrogen pickup for different oxygen activity at the bubble surface, $\mathrm{H}_{\mathrm{i}}=3 \mathrm{ppm}$, $\% \mathrm{~S}=0.02 \%, \mathrm{~m}_{\mathrm{L}}=0.097 \mathrm{~cm} \mathrm{~s}^{-1}$

23 Nitrogen pickup for different sulfur content, $\mathrm{N}_{\mathrm{i}}=30 \mathrm{ppm}, \mathrm{h}_{\mathrm{o}}=0.001$ 


\section{LIST OF FIGURES}

FIGURE

TITLE

PAGE

24 Hydrogen pickup for different sulfur concentrations at the bubble surface, $\mathrm{H}_{\mathrm{i}}=3 \mathrm{ppm}$, $\mathrm{h}_{\mathrm{o}}=0.001, \mathrm{~m}_{\mathrm{L}}=0.097 \mathrm{~cm} \mathrm{~s}^{-1}$

Nitrogen pickup for different liquid phase mass transfer coefficients, $\mathrm{N}_{\mathrm{i}}=30 \mathrm{ppm}$,

$25 \%$ S $=0.005 \%$

Hydrogen pickup for different mass transfer coefficients, $\mathrm{H}_{\mathrm{i}}=3 \mathrm{ppm}, \% \mathrm{~S}=0.02 \%$,

$26 \mathrm{~h}_{\mathrm{o}}=0.05$

Nitrogen pickup at the spout eye for different oxygen activities, $\mathrm{N}_{\mathrm{i}}=10 \mathrm{ppm}$,

$27 \% S=0.005$, stirring time $=20$ minutes, slag thickness $=5 \mathrm{~cm}$

Nitrogen pickup at the spout eye for different sulfur concentrations, $\mathrm{N}_{\mathrm{i}}=10 \mathrm{ppm}$,

$28 \mathrm{~h}_{\mathrm{o}}=0.001$, stirring time $=20$ minutes, slag thickness $=5 \mathrm{~cm}$

Nitrogen pickup at the spout eye for different stirring intervals, $\mathrm{N}_{\mathrm{i}}=10 \mathrm{ppm}$, $\% S=0.005, h_{\circ}=0.001$, slag thickness $=5 \mathrm{~cm}$ 


\section{LIST OF TABLES}

TABLE

TITLE

PAGE

\begin{tabular}{|c|c|c|}
\hline 1 & $\begin{array}{l}\text { Effect of coke addition on the hydrogen content of the liquid steel for the } \\
\text { experiment performed at Electro-Nite }\end{array}$ & 12 \\
\hline 2 & $\begin{array}{l}\text { The aluminum, oxygen, carbon and hydrogen content of the liquid iron as a } \\
\text { function of coke additions for the experiment performed at CISR laboratory - } \\
\text { Metallurgical Coke }\end{array}$ & 13 \\
\hline 3 & $\begin{array}{l}\text { The aluminum, oxygen, carbon and hydrogen content of the liquid iron as a } \\
\text { function of coke additions for the experiment performed at CISR laboratory - } \\
\text { Petroleum Coke }\end{array}$ & 13 \\
\hline 4 & Hydrogen recovery at the NSS plant trials & 15 \\
\hline 5 & $\begin{array}{l}\text { Hydrogen content of the metallurgical coke sample before and after heating to } \\
1873 \mathrm{~K}\end{array}$ & 16 \\
\hline 6 & $\begin{array}{l}\text { Hydrogen content of the petroleum coke sample before and after heating to } \\
1873 \mathrm{~K}\end{array}$ & 23 \\
\hline 7 & Average percentage recovery of nitrogen from the coke additions & 27 \\
\hline 8 & $\begin{array}{l}\text { Chemical composition (in wt percent) of the slag used in nitrogen transport } \\
\text { experiments }\end{array}$ & 28 \\
\hline 9 & Amount and type of slag-metal combination used in the transport experiments & 29 \\
\hline
\end{tabular}




\section{Executive Summary}

In recent years there has been an increasing demand to reduce and control the amount of dissolved gases in steel. Hydrogen and nitrogen are two of the most important gases which when dissolved in liquid steel affect its properties significantly. Several steelmaking additions have been investigated in this research for their effect on the hydrogen and nitrogen content of steels. It has been established that calcium hydroxide (hydrated lime) acts as a source of hydrogen. Carburizers, such as metallurgical coke, were found to result in no hydrogen pickup when added to liquid steel. Addition of petroleum coke, on the other hand, increased the hydrogen content of liquid steel. Ferroalloy such as medium carbon ferromanganese when added to the liquid iron was found to increase its nitrogen content, the increase being proportional to the amount of ferroalloy added. Similarly, addition of pitch coke, which had a significant nitrogen impurity, increased the nitrogen content of liquid iron. A mathematical model was developed to quantify the absorption of nitrogen and hydrogen from the air bubbles entrained during tapping of liquid steel. During the bottom stirring of liquid metal in a ladle, the inert gas escaping from the top displaces the slag layer and often forms an open eye. The absorption of atmospheric nitrogen through the spout eye was estimated for different slag thickness and gas flow rate.

The ultimate goal of this research was to develop a comprehensive set of equations which could predict the nitrogen and hydrogen pickup from their various sources. Estimates of hydrogen and nitrogen pickup during the steel transfer operations such as tapping and ladle stirring and the predicted pickup from steelmaking additions were integrated into empirical equations. The comprehensive model is cesigned to predict the gas pickup under varying operating conditions such as the metal oxygen and sulfur content, the total tapping or stirring time, the stirring gas flow rate and the slag thickness. The model predictions are based on mathematical and empirical evidence which are derived from thermodynamic and kinetic fundamental principles. 


\section{Introduction}

Control of hydrogen and nitrogen content of steels during steelmaking has become important during the recent years. Nitrogen in steel can be in its uncombined form as free nitrogen or in the form of a compound or nitride. Uncombined nitrogen dissolves interstitially in steel and increases the yield and tensile strength of the metal due to the interaction of carbon and nitrogen atoms with the dislocations (strain aging). During solidification, the dissolved nitrogen may react with $A$ l to form nitrides (AIN), which reduces the formability of the steel. Steel from an Electric Arc Furnace (EAF) normally has higher nitrogen levels (70-120 ppm) compared to that produced in a Basic Oxygen Furnace (BOF) where nitrogen varies between 20 and 60 ppm. Hence, nitrogen is of particular importance in an EAF plant. Hydrogen has been recognized as being detrimental to steel. It may lead to problems like hydrogen embrittlement, hydrogen blistering and hairline cracks (flakes). These affect the mechanical properties and lead to the premature failure of steel. At high concentrations, hydrogen and nitrogen can evolve during solidification and cause porosity in ingots and castings. Control of hydrogen and nitrogen is therefore of prime concern in steelmaking.

Numerous sources of hydrogen and nitrogen exist during the melting, the ladle processing and the casting operations. Sources of nitrogen in oxygen steelmaking (OSM) include the hot metal, the scrap, the impurity nitrogen in oxygen and the nitrogen used as a stirring gas. Nitrogen pickup from the atmosphere can occur during reblows in which case the furnace fills up with air, which is then entrained into the metal when the oxygen blow restarts. Also during the tapping of steel, air bubbles are entrained into the steel where the tap stream enters the bath in the ladle. Other sources may include atmosphere (through ladle slag), coke (carburizers) and various ferro alloys. Ladle additions often contain moisture. Hydrogen pickup in the steel is primarily due to the water associated with the slagmaking materials and as an impurity in the alloy additions and the carburizers. Hydrogen is generally not a problem in the OSM except in the bottom blown converters (Q-BOP) where natural gas $\left(\mathrm{CH}_{4}\right)$, used as a tuyere coolant, is the major source of hydrogen. Vacuum degassing can remove hydrogen but it is not very effective in the case of nitrogen where removal is normally less than $20 \%$. During 
most ladle treatments there is a significant nitrogen pickup from the atmosphere. Therefore other ways to reduce the hydrogen and nitrogen in the steel are to be sought.

Possibilities of removing nitrogen by using the slag have been explored and a considerable research has been done on the nitride capacity of slags. As discussed elsewhere, slags cannot remove significant amounts of nitrogen economically. It is generally thought that the slag insulates the melt surface from the atmosphere. However, nitrogen transfer from the atmosphere through the slag layer may result in a significant nitrogen pickup in the melt. Since it is not possible to remove hydrogen and nitrogen without vacuum degassing, the pickup of these elements in the ladle and casting operations must be minimized.

\section{Objective}

The removal of dissolved hydrogen and nitrogen from steel is difficult and costly. It is necessary, therefore, to understand the sources of these gases in order to keep their pickup during steelmaking to a minimum. The objective of this research is to identify and quantify the sources of hydrogen and nitrogen during tapping and ladle operations. This information will then be used to develop a comprehensive model that can predict and hence aid in the controlling the final content of these dissolved gases.

\section{Research Results}

The results are summarized below and details are given in Appendix A.

\section{Task I. Hydrogen pickup from $\mathrm{Ca}(\mathrm{OH})_{2}$}

$\mathrm{CaO}$ is an important addition during steelmaking operations, especially during ladle metallurgy. Hydrated lime which exists as $\mathrm{Ca}(\mathrm{OH})_{2}$ was found to be a source of hydrogen. Under experimental conditions in this study, about 2 percent of the hydrogen present in the hydroxide was found to go into liquid metal. This low value is possibly because $\mathrm{Ca}(\mathrm{OH})_{2}$ has a decomposition temperature of $811 \mathrm{~K}$ which is low as compared to steelmaking temperature of more than $1800 \mathrm{~K}$. Consequently most of the water vapor 
dissociated from the hydroxide escapes into the atmosphere before it could further dissociate and enter as hydrogen into the melt.

\section{Task II. Hydrogen and Nitrogen Pickup from Alloys and Carburizers}

Addition of metallurgical coke, having close to $0.2 \%$ hydrogen content, to medium carbon steel did not result in any hydrogen pickup by the melt. A possible explanation is the loss of more than $40 \%$ of the hydrogen in the form of volatiles upon heating this coke to steelmaking temperatures. Petroleum coke, on the other hand, caused a hydrogen pickup proportional to the amount of coke addition. A total hydrogen recovery of about $10 \%$ was registered from these additions. Ferroalloys which are added to modify the composition of the liquid steel have inherent nitrogen impurity. Experiments were performed to determine the effect of medium carbon ferromanganese on the nitrogen content of pure iron. It was observed that the nitrogen content of the melt increased after each ferroalloy addition. All the nitrogen present in the ferroalloy was found to result into the melt. This is probably because nitrogen is present atomically in the ferroalloy therefore the rate limiting step of dissociation of the gaseous nitrogen molecule is not present.

Another possible source of nitrogen is the impurity nitrogen associated with the coke additions. In the laboratory, experiments were performed where pitch coke was added to liquid metal. It was found that the nitrogen content of the melt increased following each coke addition and an average nitrogen recovery of $20 \%$ nitrogen was observed from these additions. Some of this dissolved nitrogen was found to desorb towards the end of the experiment and it was speculated that such desorption occurred throughout the experiment. It was experimentally verified that the nitrogen content of the coke decreased on heating it to steelmaking temperatures of $1550{ }^{\circ} \mathrm{C}$. As a result only the nitrogen present as a compound, possibly in the form of nitrides or cyanides, entered the liquid metal while the remainder escaped as a gaseous species during the course of heating. 


\section{Task III. Transport of Nitrogen through Slag}

The presence of a slag layer on top of the liquid steel is generally believed to insulate it from the atmospheric gases. Experiments were performed using a resistance furnace where a liquid slag-metal system was maintained under a nitrogen atmosphere. Transport of nitrogen from the atmosphere to the metal through the slag layer was studied for various lengths of time and was found to be very slow. Fick's Second Law was used to estimate the diffusion of nitrogen through the slag layer. The predictions from the diffusion model agreed with the experimental results.

\section{Task IV. Model for Ladle Processing}

\section{Nitrogen and Hydrogen Pickup during Tapping}

Mathematical models were developed to predict the hydrogen and nitrogen pickup during the tapping of liquid steel. This pickup occurs from the air bubble entrainment during the tapping. Computational Fluid Dynamic software, CFX 5.5.1, was used to compute the total interfacial area of the bubbles in contact with the liquid metal. Mixed control models, incorporating liquid phase mass transfer and chemical kinetics in series, were then used to calculate the hydrogen and nitrogen pickup for different operating conditions. These models were found to predict the hydrogen and nitrogen pickup values ranging from 0.5 to $8 \mathrm{ppm}$ and 8 to $40 \mathrm{ppm}$ respectively for different oxygen and sulfur content in the metal and partial pressure of water vapor in the atmosphere.

A physical tapping model was developed to validate the results of the CFD model developed to estimate gaseous pickup during tapping. $\mathrm{A} \mathrm{CO}_{2}$ - aqueous $\mathrm{NaOH}$ solution technique was used to simulate nitrogen absorption during the tapping of liquid steel. Reasonable values of the liquid phase mass transfer coefficient were estimated using the results of the physical and the CFD model which were helpful in validating the CFD model. Comparison was also made with an industrial data and the results were found to agree within reasonable limits. 
During bottom gas stirring in a ladle metallurgical operation, nitrogen pickup can take place at the spout eye. A mathematical model was developed to calculate the extent of nitrogen absorption at the spout eye. Inputs to this model included the spout eye area and the liquid phase mass tansfer coefficient at the plume eye. Empirical correlations, from existing research, were used to compute the area of the exposed metal. The mass transfer coefficient was estimated using the surface renewal theory. The surface renewal velocity, needed for mass transfer calculations, was obtained from CFD calculations performed using CFX 5.6. The model calculations demonstrated that a lower oxygen and sulfur concentrations, a higher gas flow rate, presence of a thinner slag layer and a longer stirring time are the favorable conditions for a higher nitrogen pickup.

\section{Task V. Model for Ladle and Tapping Operations}

A comprehensive model has been compiled based on the findings of various experimental results and models developed in this research. This model includes a set of mathematical equations which can be used to estimate the hydrogen and nitrogen pickup from the various sources investigated in this research. An example of the predictions of hydrogen and nitrogen pickup for a set of operating conditions using the comprehensive equations is given in the table below. 


\section{Comprehensive model to predict total hydrogen and nitrogen pickup during tapping and ladle operations}

Weight of metal $(\mathrm{kg})=$

Oxygen content of metal $(\mathrm{wt} \%)=$

Sulfur content of metal $(w t \%)=$

$\mathrm{H}_{2} \mathrm{O}$ partial pressure $(\mathrm{atm})=$

Weight of lime $(\mathrm{kg})=$

Weight of ferroalloy added $(\mathrm{kg})=$

Nitrogen impurity in the alloy $(w t \%)=$

Weight of Coke added $(\mathrm{kg})=$

Nitrogen impurity in coke $(w t \%)=$

Hydrogen impurity in coke $(w t \%)=$

Stirring time $(\min )=$

Bottom gas flow rate $($ SCFM) $=$

Slag layer thickness $(\mathrm{cm})=$

Hydrogen pickup from additions (ppm) = Hydrogen pickup during tapping $(\mathrm{ppm})=$

Nitrogen pickup from additions $(\mathrm{ppm})=$ Nitrogen pickup during tapping $(\mathrm{ppm})=$ Nitrogen pickup during stirring $(\mathrm{ppm})=$

Total Hydrogen pickup $(\mathrm{ppm})=$

Total Nitrogen pickup $(p p m)=$
200000

0.05

0.02

0.03

200

50

0.1

100

1

0.3

17

10

7

0.69

1.50

1.25

14.64

0.61

2.19

16.49 


\section{Appendix A}

\section{Task I Hydrogen Pickup from $\mathrm{Ca}(\mathrm{OH})_{2}$}

Small-scale industrial trials were performed to investigate the effect of calcium hydroxide addition on the hydrogen content of medium carbon steels. Figure 1 shows the hydrogen pickup as a function of time for the first experiment. In the first trial the $\mathrm{CaO}-\mathrm{Al}_{2} \mathrm{O}_{3}$ slag was added on top of the liquid metal. The variation of the hydrogen content as a function of $\mathrm{Ca}(\mathrm{OH})_{2}$ addition is plotted for the second trial in Figure 2. In this case the calcium hydroxide was added along with the slag. Also shown in both the figures is the oxygen content as a function of time during the experiment.

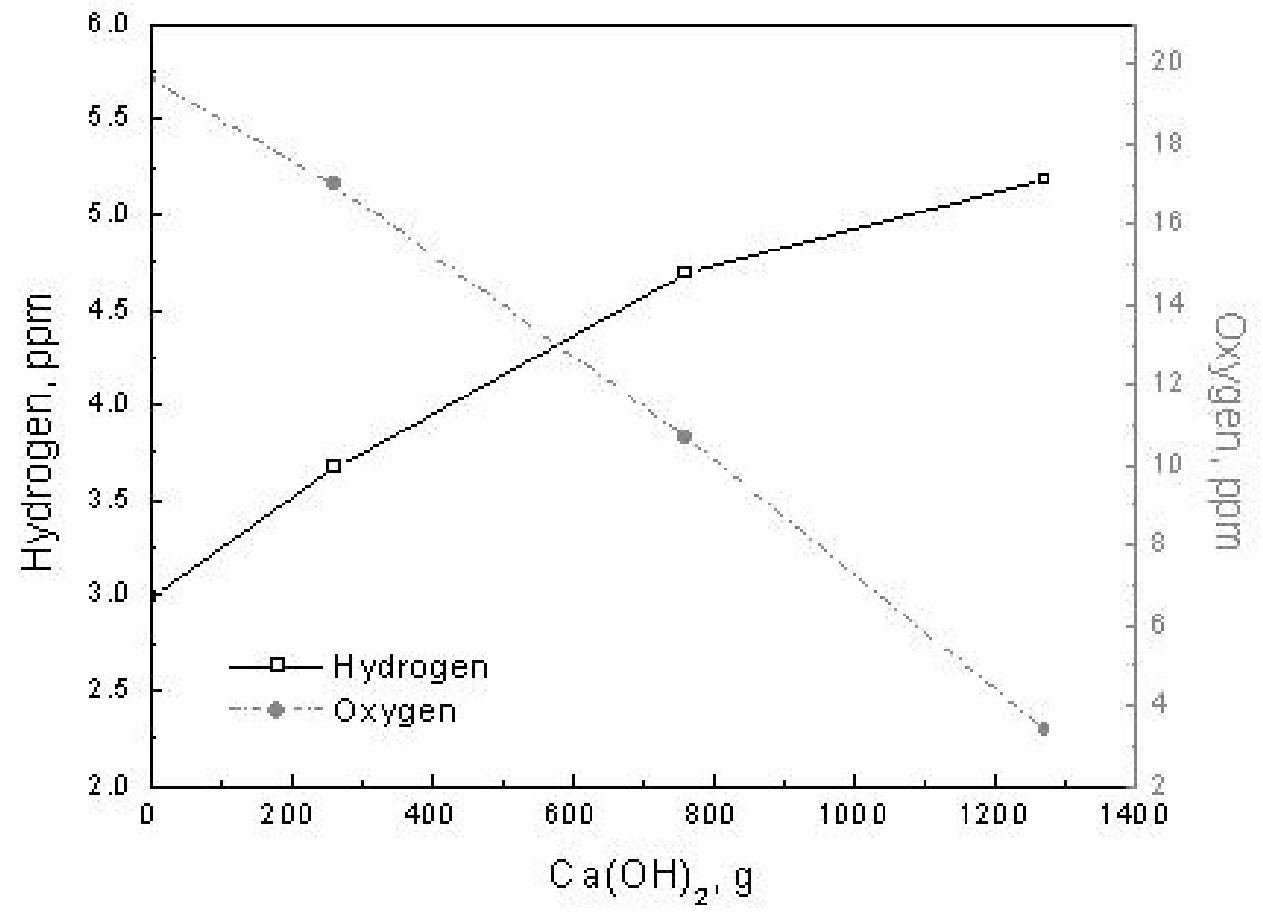

Figure 1, Hydrogen pickup due to $\mathrm{Ca}(\mathrm{OH})_{2}$ addition on top of the slag

\section{Discussion}

Hydrogen pickup in steel primarily occurs from the dissociation of water. Calcium oxide is added to adjust slag chemistry, to facilitate inclusion removal and for 
desulfurization during the secondary steelmaking. Due to the moist atmospheric conditions, lime can become hydrated to form calcium hydroxide. This hydrated lime when added to the liquid melt decomposes according to the reaction

$$
\mathrm{Ca}(\mathrm{OH})_{2}(\mathrm{~s})=\mathrm{CaO}(\mathrm{s})+\mathrm{H}_{2} \mathrm{O}(\mathrm{g}) \quad \text { Equation } 1
$$

The water vapor formed dissociates on the liquid steel surface causing hydrogen pickup by the following reaction

$$
\mathrm{H}_{2} \mathrm{O}(\mathrm{g})=2[\mathrm{H}]+[\mathrm{O}] \quad \text { Equation } 2
$$

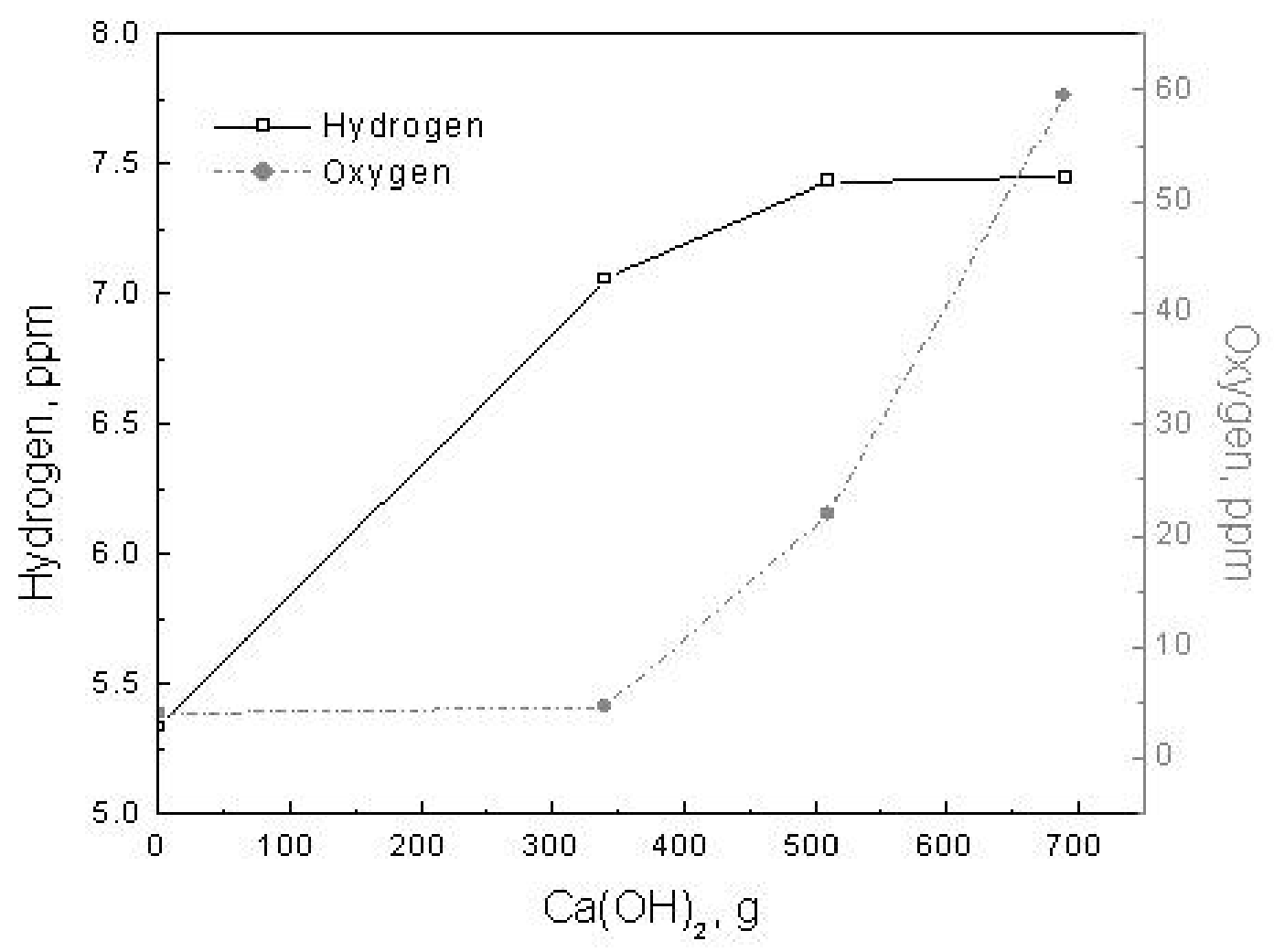

Figure 2, Hydrogen pickup due to $\mathrm{Ca}(\mathrm{OH})_{2}$ addition along with the slag

The previous reaction is thermodynamically favorable for deoxidized steel where substantial hydrogen pickup can occur [1]. It can be seen that for the first trial the 
hydrogen content increases with each addition of $\mathrm{Ca}(\mathrm{OH})_{2}$. A total of $1260 \mathrm{~g}$ of calcium hydroxide was added to the melt which supplies 34 moles of hydrogen. If all of this hydrogen were to dissolve in the melt, then by mass balance calculations the total hydrogen content would have increased to a hypothetical value of $150 \mathrm{ppm}$. However, an increase of only $2.2 \mathrm{ppm}$, which is less than $1.5 \%$ of the added amount, was observed. For the second experiment, $690 \mathrm{~g}$ of calcium hydroxide, equivalent to 19 moles of hydrogen, was added. An increase of $2.1 \mathrm{ppm}$ was observed. This represents only about $2 \%$ of the total hydrogen added as $\mathrm{Ca}(\mathrm{OH})_{2}$.

From Figure 1 it can be seen that the hydrogen pickup is proportionate to the amount of $\mathrm{Ca}(\mathrm{OH})_{2}$ added. This is not the case in the second experiment (Figure 2). Possible reasons for this observation are that in the latter case, the melt had an increasing oxygen level and a fairly high starting hydrogen content of $5.3 \mathrm{ppm}$. The driving force for further absorption of the hydrogen was considerably lower once the hydrogen content reached $7 \mathrm{ppm}$ after the first addition of $\mathrm{Ca}(\mathrm{OH})_{2}$. This can be explained by the following rate equation, which assumes a liquid phase mass transfer control for the absorption of hydrogen in the liquid iron:

$$
\ln \left(\frac{[\% H]_{t}-[\% H]_{e}}{[\% H]_{i}-[\% H]_{e}}\right)=-k_{H} t \quad \text { Equation 3 }
$$

where $[\% \mathrm{H}]_{\mathrm{t}},[\% \mathrm{H}]_{\mathrm{e}}$ and $[\% \mathrm{H}]_{\mathrm{i}}$ are the instantaneous, equilibrium and initial hydrogen contents, $\mathrm{k}_{\mathrm{H}}$ is the liquid phase mass transfer constant and $\mathrm{t}$ is the time.

The capacity of the melt to absorb the hydrogen decreases as the hydrogen content in the melt approaches the equilibrium value. The capacity to absorb hydrogen increases as the steel is deoxidized. A similar case is observed in the second experiment, Figure 2, where the equilibrium hydrogen decreases as the oxygen content increases. It is found that more hydrogen is absorbed when the calcium hydroxide is added along with the slag as opposed to when it is added on top of it. The decomposition of $\mathrm{Ca}(\mathrm{OH})_{2}$ takes place according to Equation 1 and the equilibrium constant and the Gibbs free energy [2] for that reaction are given as follows 


$$
\begin{array}{cr}
K=\frac{1}{p_{\mathrm{H}_{2} \mathrm{O}}} & \text { Equation } 4 \\
\Delta G^{o}=-117600+145 T\left(\mathrm{~J} \mathrm{~mol}^{-1}\right) & \text { Equation } 5
\end{array}
$$

For hydrogen to dissolve in the liquid metal, the partial pressure of the water vapor must reach unity. Using the above equations it is found that $\mathrm{p}_{\mathrm{H}_{2} \mathrm{O}}=1$ for $\mathrm{T}=811 \mathrm{~K}$. The hydroxide dissociates at a temperature much lower than the steelmaking temperature. Therefore when $\mathrm{Ca}(\mathrm{OH})_{2}$ is added on top of the slag, it is likely that most of the water vapor escapes into the atmosphere before it could react with the liquid metal. In actual steelmaking operations, lime is added along with the other slag making material hence trial 2 is of more relevance.

Table 1, Effect of coke addition on the hydrogen content of the liquid steel for the experiment performed at Electro-Nite

\begin{tabular}{|c|c|c|c|c|}
\hline Coke addition $(\mathrm{g})$ & \multicolumn{2}{|c|}{ Before coke addition } & \multicolumn{2}{c|}{ After coke addition } \\
\hline & Oxygen, ppm & Hydrogen, ppm & Oxygen, ppm & Hydrogen, ppm \\
\hline 0 & 5.7 & 3.8 & - & - \\
\hline 1140 & 59.5 & $\mathbf{6 . 2 8}$ & 5.2 & $\mathbf{4 . 8 4}$ \\
\hline 1140 & 4.3 & $\mathbf{6 . 6 8}$ & 13.7 & $\mathbf{6 . 0 7}$ \\
\hline 1140 & 8.0 & $\mathbf{8 . 2 8}$ & 6.2 & $\mathbf{6 . 8 6}$ \\
\hline
\end{tabular}

\section{Task II Hydrogen and Nitrogen Pickup from Alloys and Carburizers}

\section{(a) Hydrogen Pickup}

\section{Metallurgical Coke}

The effect of metallurgical coke addition on the hydrogen content of low carbon steel was investigated in a trial at Electro-Nite. The hydrogen partial pressure values measured by $\mathrm{HYDRIS}^{\circledR}$ were used to obtain the actual hydrogen concentration. Table 1 
shows the hydrogen content before and after each coke addition. The oxygen content at each hydrogen measurement is also shown. In a laboratory scale experiment, it was possible to start with a relatively lower level of hydrogen. Table 2 shows the results of the coke addition on the hydrogen and carbon content of the metal.

Table 2, The aluminum, oxygen, carbon and hydrogen content of the liquid iron as a function of coke additions, experiment performed at CISR laboratory

\begin{tabular}{|c|c|c|c|c|}
\hline Time, minutes & Al, wt \% & Oxygen, ppm & Carbon, wt \% & Hydrogen, ppm \\
\hline 0 & 0.031 & 27 & 0.002 & $\mathbf{1 . 1 6}$ \\
\hline 7 & - & - & 0.034 & $\mathbf{1 . 2 0}$ \\
\hline 15 & - & - & 0.170 & $\mathbf{0 . 8 1}$ \\
\hline 25 & - & 23 & 0.075 & $\mathbf{1 . 2 3}$ \\
\hline
\end{tabular}

\section{Petroleum Coke}

A series of plant trials were performed at North Star Steel where varying amount of coke was added to different heats of 90 tonne liquid steel in a Ladle Refining Station (LRS). Three hydrogen measurements were subsequently made in the liquid metal. The first measurement $(\mathrm{H} 1)$ was when the liquid metal reached the ladle refining station (LRS), the second measurement (H2) was after all the additions were made and the ladle was about to leave the LRS and the third one $(\mathrm{H} 3)$ was made in the tundish. The hydrogen pickup due to coke additions at the LRS is the difference in the first and the second measurement and is shown in Figure 3.

Table 3, The Aluminum, oxygen, carbon and hydrogen content of the liquid iron as a function of coke additions, experiment performed at CISR laboratory

\begin{tabular}{|c|c|c|c|c|c|}
\hline Sample \# & Time, min & Al, wt\% & Oxygen, ppm & Carbon, wt\% & Hydrogen, ppm \\
\hline S1 & 0 & 0.14 & 40 & 0.001 & 1.47 \\
\hline S2 & 2 & - & - & - & 1.25 \\
\hline S3 & 30 & - & - & 0.06 & 2.84 \\
\hline S4 & 60 & - & - & 0.21 & 1.92 \\
\hline \multicolumn{7}{|c|}{ Recovery from the first coke addition } \\
\hline
\end{tabular}


As a follow up of these trials, one more experiment was performed at CMU with the objective of starting at lower hydrogen content. Table 3 shows the hydrogen pickup as a function of coke addition for that experiment. In order to make a fair comparison the percentage recovery of hydrogen from the coke addition was calculated for both the plant trials and the laboratory experiment. Table 4 shows the recovery of hydrogen in each heat for the NSS trials.

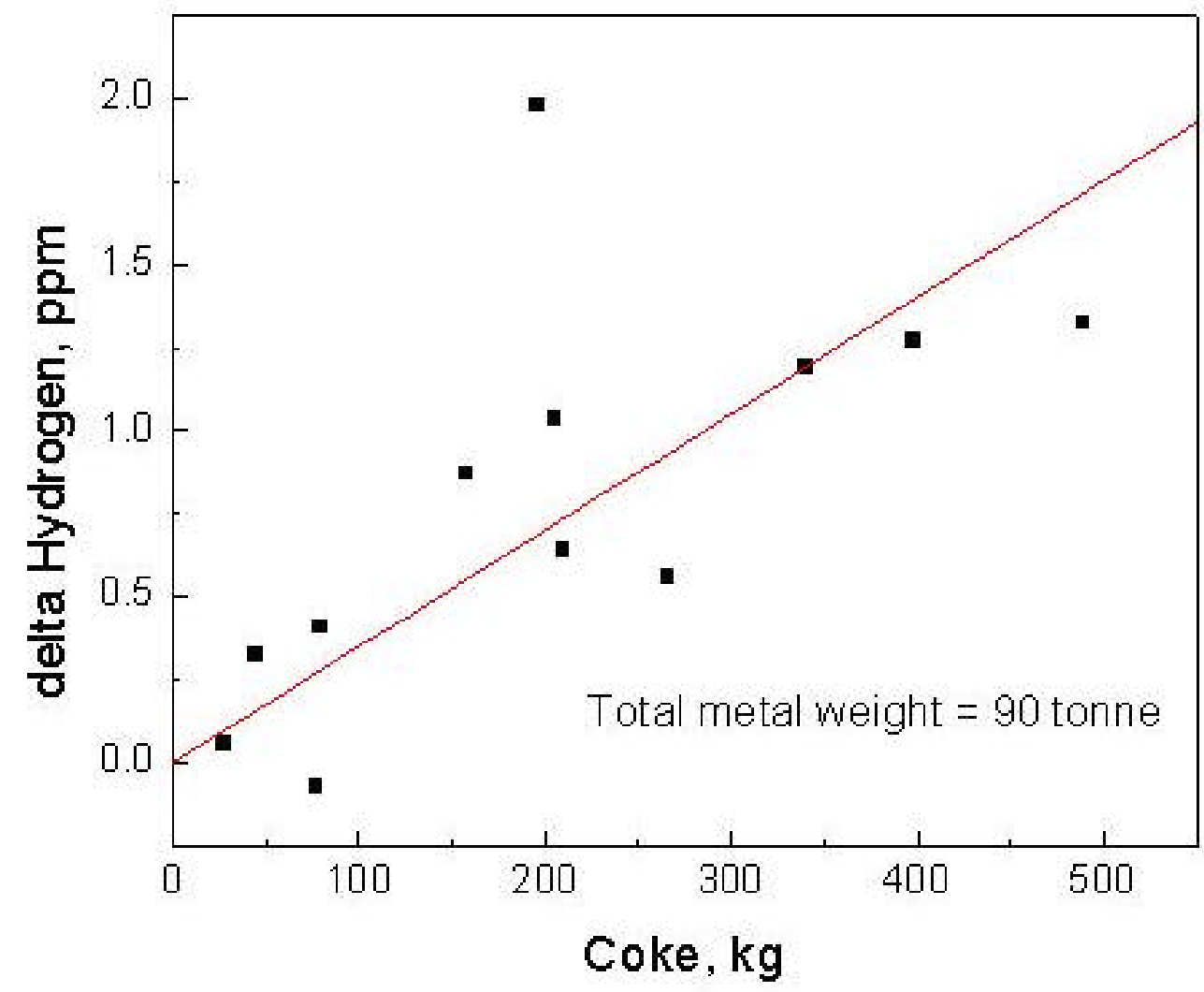

Figure 3, Change in the hydrogen content of the liquid metal (at the LRS) as a function of coke addition for 90 tonne heat

\section{Discussion}

\section{For Metallurgical Coke Addition}

The hydrogen content of the metallurgical coke used in these experiments was reported as $0.20 \%$. It is seen from Table 1 that the hydrogen content of steel decreases after each coke addition. It is difficult to theorize a scientific explanation for the observed 
decrease of hydrogen after addition of coke. One of the possible explanations could be the excessive HYDRIS ${ }^{\circledast}$ usage and frequent probing of the melt. It is worth mentioning that at the time of this experiment, different kinds of $\mathrm{HYDRIS}^{\circledast}$ probes were being simultaneously tested. It was later verified that these different probes obtained consistent readings hence no correction was needed in this regard.

Table 4, Hydrogen recovery at the NSS plant trials

\begin{tabular}{|c|c|c|c|c|}
\hline Coke $(\mathrm{kg})$ & $\begin{array}{c}\text { Total Hydrogen } \\
\text { added }(\mathrm{kg})\end{array}$ & $\begin{array}{c}\text { Hydrogen } \\
\text { added [ppm] }\end{array}$ & $\begin{array}{c}\text { Hydrogen pickup } \\
\text { at LRS [ppm] }\end{array}$ & $\begin{array}{c}\% \\
\text { recovery }\end{array}$ \\
\hline 27.24 & 0.082 & 0.91 & 0.05 & 6.05 \\
\hline 44.49 & 0.133 & 1.48 & 0.32 & 21.69 \\
\hline 77.18 & 0.232 & 2.57 & -0.07 & -2.91 \\
\hline 78.99 & 0.237 & 2.63 & 0.41 & 15.58 \\
\hline 157.54 & 0.473 & 5.25 & 0.87 & 16.59 \\
\hline 196.13 & 0.588 & 6.54 & 1.98 & 30.28 \\
\hline 205.21 & 0.616 & 6.84 & 1.04 & 15.19 \\
\hline 209.75 & 0.629 & 6.99 & 0.64 & 9.11 \\
\hline 265.59 & 0.797 & 8.85 & 0.56 & 6.29 \\
\hline 339.59 & 1.019 & 11.32 & 1.19 & 10.53 \\
\hline 398.16 & 1.194 & 13.27 & 1.27 & 9.58 \\
\hline 489.41 & 1.468 & 16.31 & 1.32 & 8.10 \\
\hline \multicolumn{5}{|c|}{ Average recovery } \\
\hline
\end{tabular}

Table 2 shows the result of coke addition in a laboratory scale experiment which was conducted in a controlled inert atmosphere. It was important to maintain low oxygen content in the metal because of its effect on hydrogen absorption in liquid iron. For the same reason, the liquid metal was deoxidized by aluminum addition and the oxygen content of the liquid metal was maintained close to $25 \mathrm{ppm}$ during the experiment. The results suggest that the first two of the three coke additions were successful since the carbon content was found to increase. The hydrogen content, however, was found to remain close to its starting value of $1 \mathrm{ppm}$. Given the limitations of the sampling technique it can be said that very little of the hydrogen in the coke entered the steel. 
It is our hypothesis that hydrogen is released as a gas when the coke particles are heated and such a release takes place at temperatures lower than steelmaking temperature. Due to the kinetic and mass transfer limitations, very little of this hydrogen gas enters the steel. In order to confirm this hypothesis, it was planned to heat the coke samples to steelmaking temperature and record the change in the hydrogen content. For such a test, a known weight of metallurgical coke sample was pulverized to -60 mesh. The first half of this sample was heated to $1823 \mathrm{~K}$ in an argon atmosphere for 6 hours and the other half was stored in an atmosphere free of moisture. Both these samples were then sent for hydrogen analysis to Commercial Test and Engineering, South Holland - II and the results are shown in Table 5. From these results, it can be seen that more than $40 \%$ of the hydrogen present in the metallurgical coke is lost upon heating to the steelmaking temperature. For the same reason, no hydrogen pickup was observed from the metallurgical coke experiments.

Table 5, Hydrogen content of the metallurgical coke sample before and after heating to $1873 \mathrm{~K}$

\begin{tabular}{|c|c|c|c|}
\cline { 2 - 4 } \multicolumn{1}{c|}{} & Hydrogen as received & Hydrogen Dry basis & Moisture \\
\hline Coke, as received & 0.55 & 0.55 & 0.50 \\
\hline Coke, heated to $1550{ }^{\circ} \mathrm{C}$ & 0.32 & 0.32 & - \\
\hline
\end{tabular}

\section{For Petroleum Coke Addition}

From Figure 3 it is clear that unlike the case of metallurgical coke, addition of petroleum coke causes an increase in the hydrogen content. As a general trend, it is also noticed that a higher pickup is observed for a larger coke addition. Similar hydrogen increase is found from the results of the laboratory experiment shown in Table 3. When comparing the hydrogen measured for sample $\mathrm{S} 1$ and $\mathrm{S} 3$, a pickup of $1.5 \mathrm{ppm}$ was noticed. However, no hydrogen pickup was noticed after the second coke addition (difference between S4 and S3) even though the carbon level was found to increase. This indicates that the coke addition was successful and a lower hydrogen value can be attributed to the sampling error. To determine the effect of sampling technique on the hydrogen measurement two hydrogen samples were taken in quick succession of each 
other. The first two measurements (S1 and S2), shown in Table 3, illustrate the difference. There is a higher hydrogen content registered in the first sample (S1), which was taken with more caution than the second one (S2), thereby showing that any negligence in the sampling technique causes hydrogen loss possibly due to diffusion.

Table 4 shows an average recovery of $12 \%$ for the NSS trials as opposed to $8 \%$ in the laboratory experiment. These values are considered to be in good agreement with each other. The difference between these is primarily due to sampling errors involved in the small scale experiment. It is noticed that chemical analysis normally measures lower hydrogen value than $\mathrm{HYDRIS}^{\circledR}$ with the difference being close to a factor of 2 . As in the case of metallurgical coke, the change in the hydrogen content on heating the coke sample was analyzed for the petroleum coke as well. The hydrogen contents of the two samples, before and after heating to $1823 \mathrm{~K}$, are shown in Table 6 . In this case, however, no hydrogen decrease was seen after heating the coke sample to steelmaking temperature. This can possibly explain a hydrogen pickup on addition of petroleum coke against no such increase on addition of metallurgical coke.

Table 6, Hydrogen content of the petroleum coke sample before and after heating to $1873 \mathrm{~K}$

\begin{tabular}{|c|c|c|c|}
\cline { 2 - 4 } \multicolumn{1}{c|}{} & Hydrogen as received & Hydrogen Dry basis & Moisture \\
\hline Coke, as received & 0.23 & 0.23 & 0.55 \\
\hline Coke, heated to $1550^{\circ} \mathrm{C}$ & 0.22 & 0.22 & - \\
\hline
\end{tabular}

\section{(b) Nitrogen Pickup}

\section{Effect of Ferroalloy Addition}

In the steelmaking process route, some ferroalloys such as Fe-Si and Fe-Mn are added during tap [3]. The ferroalloy investigated in this research was medium carbon ferromanganese. Figure 4 shows the experimental nitrogen pickup as a function of ferromanganese addition for the first trial. Also shown in the same figure is the expected nitrogen pickup based on mass balance calculations. Results for the next trial are shown 
in Figure 5. In this case the pickup is shown as a function of time; amount of ferroalloy added was equal to $20 \mathrm{~g}$ for each addition. As in the first case, nitrogen pickup expected from mass balance calculations is also shown.

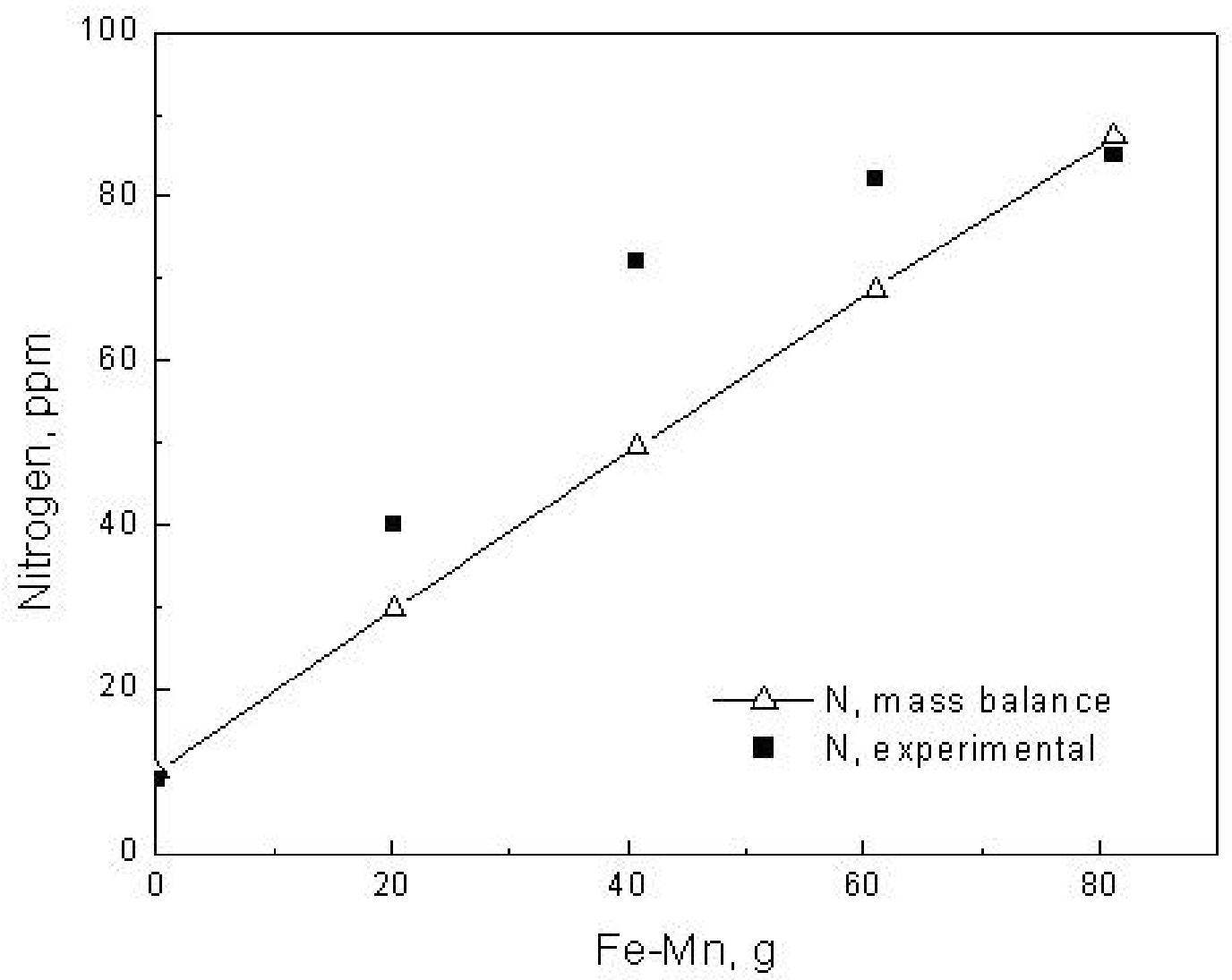

Figure 4, Nitrogen pickup from medium carbon ferromanganese, Trial 1

\section{Discussion}

For both the experiments, remarkably low initial nitrogen content of $10 \mathrm{ppm}$ could be achieved due to the controlled atmospheric conditions. The mass balance value is found to under predict (Trial 1) or over predict (Trial 2) the experimental value. Two possible reasons for the deviation could be

1. The absorption of atmospheric nitrogen

2. The inconsistency of the nitrogen content in the ferromanganese. 


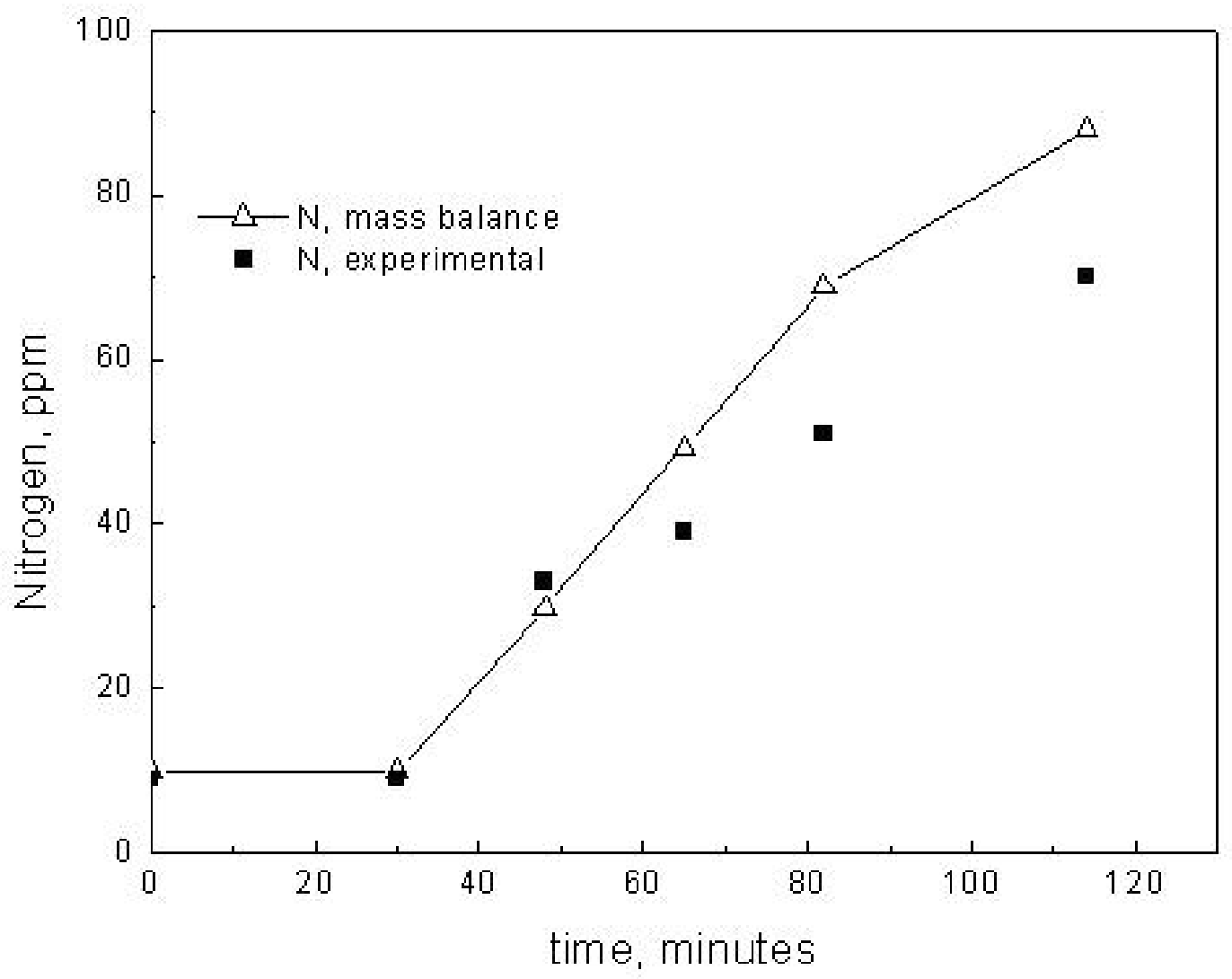

Figure 5, Nitrogen pickup from medium carbon ferromanganese, Trial 2

To verify the first possibility, in the second trial an additional metal sample was taken before any ferroalloy addition. It was found that the nitrogen content of the melt remained constant over a span of 30 minutes. This proves that the atmospheric nitrogen did not contribute to the nitrogen pickup in the melt. To explore the second possibility a few randomly chosen pieces of ferromanganese were sent for chemical analysis to verify their impurity nitrogen content. The results of this analysis showed that the impurity content varied from $0.04 \%$ to $0.11 \%$ nitrogen for different pieces of the ferroalloy. The average nitrogen impurity content was close to the initially reported value of $0.1 \%$. It can be said that not all the ferroalloy pieces had the same nitrogen impurity content. The positive or negative deviation from the theoretical results based on mass balance is due to the addition of ferromanganese pieces having a range of nitrogen impurity content. It 
can therefore be concluded that all the nitrogen from the ferromanganese results into the melt.

\section{Effect of Pitch Coke Addition}

In this part of the research, a few experiments were performed to determine if carburizers act as a source of nitrogen. In the first experiment, 4 coke additions of $50 \mathrm{~g}$ each were made at a regular interval of 17 minutes. The experimental plot for carbon and nitrogen pickup as a function of coke addition is shown in Figure 6. It is seen that the starting nitrogen content was a high of $133 \mathrm{ppm}$ because of some nitrogen pickup from an exposed spout eye due to strong stirring. The nitrogen content did not show a consistent trend in its increase or decrease.

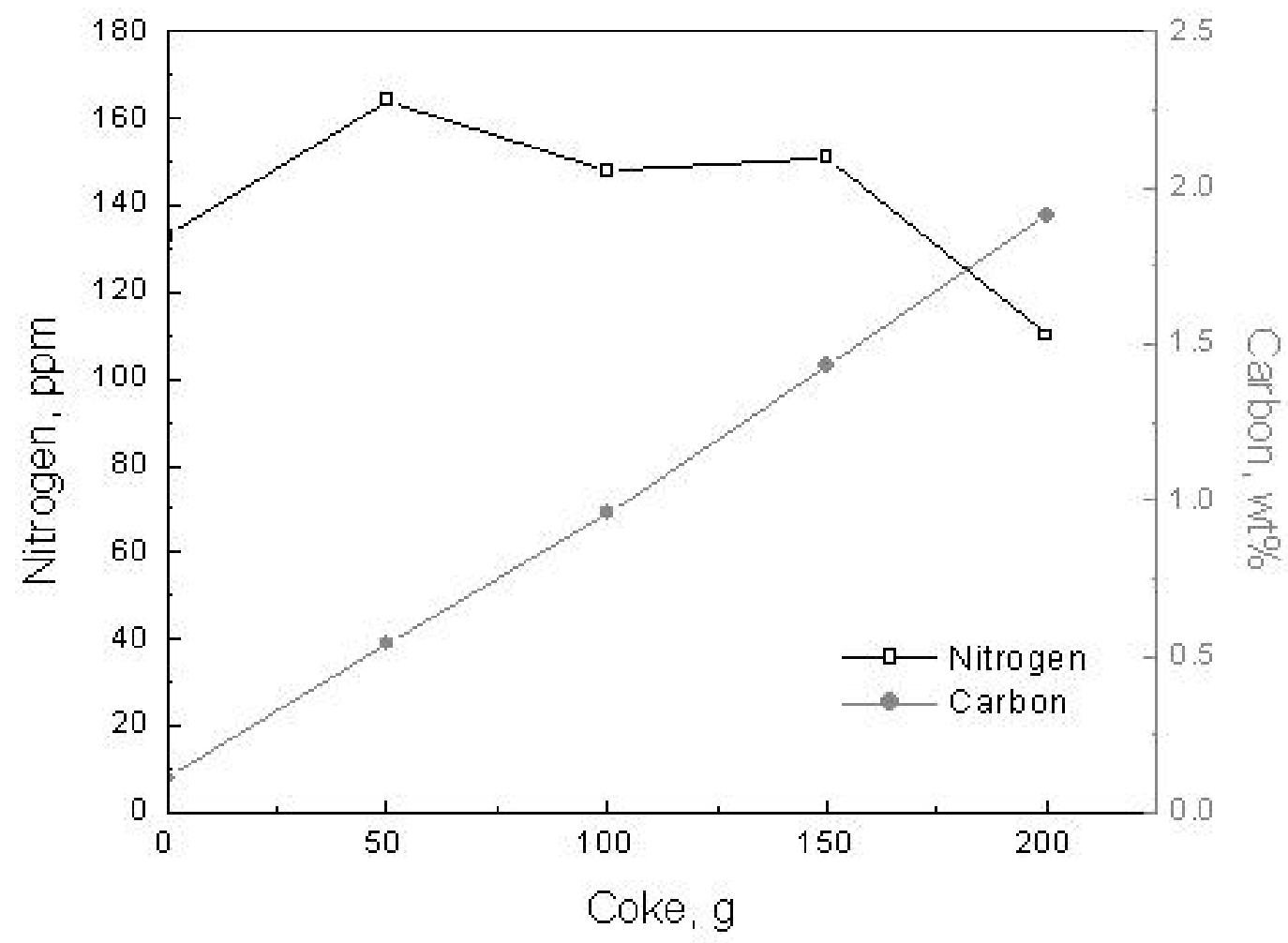

Figure 6, Effect of pitch coke addition on the nitrogen and carbon content of iron, Trial 1 
To obtain a better understanding of the actual process, two more trials were performed with the aim of starting with lower nitrogen content. In these trials, each addition consisted of $35 \mathrm{~g}$ of coke. Metal samples were taken 10 minutes after each such addition. The results for these experiments are shown in Figure 7 and Figure 8 . It is seen that in both these cases the starting nitrogen content is slightly lower and a steady increase in the nitrogen pickup is seen with each coke addition. However, towards the end of Trial 2, the nitrogen content stays almost constant. To confirm the effect of atmospheric nitrogen, in Trial 3 an additional metal sample was taken before the first coke addition. The nitrogen concentration was found to be constant which meant that atmospheric nitrogen was not being absorbed. An additional sample was also taken after the last coke addition and it was found that nitrogen was desorbing from the melt.

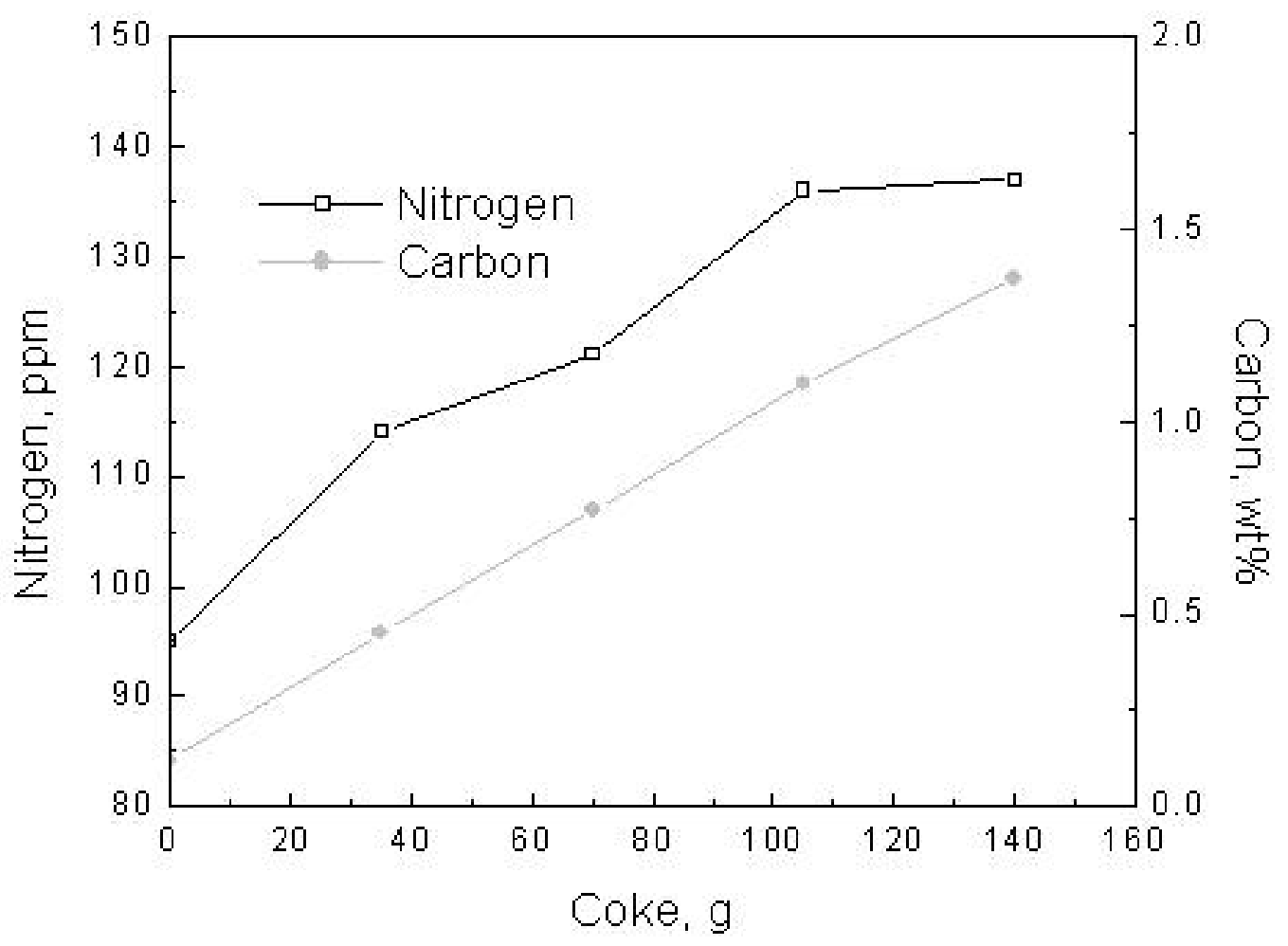

Figure 7, Effect of pitch coke addition on the nitrogen and carbon content of iron, Trial 2 
All the previous trials were performed for inductively heated melts with a poor atmospheric control. The next trial in this task was performed in a resistance furnace. The effect of coke addition, each addition being equal to $3.5 \mathrm{~g}$, is shown in Figure 9 . Remarkably enough, the starting nitrogen content was lower than $10 \mathrm{ppm}$. The carbon content was found to increase with each coke addition suggesting thereby that coke in the form of carbon was going into solution. In accordance with the previous trial no atmospheric nitrogen was found to enter the liquid metal and nitrogen desorption was observed towards the end of the experiment.

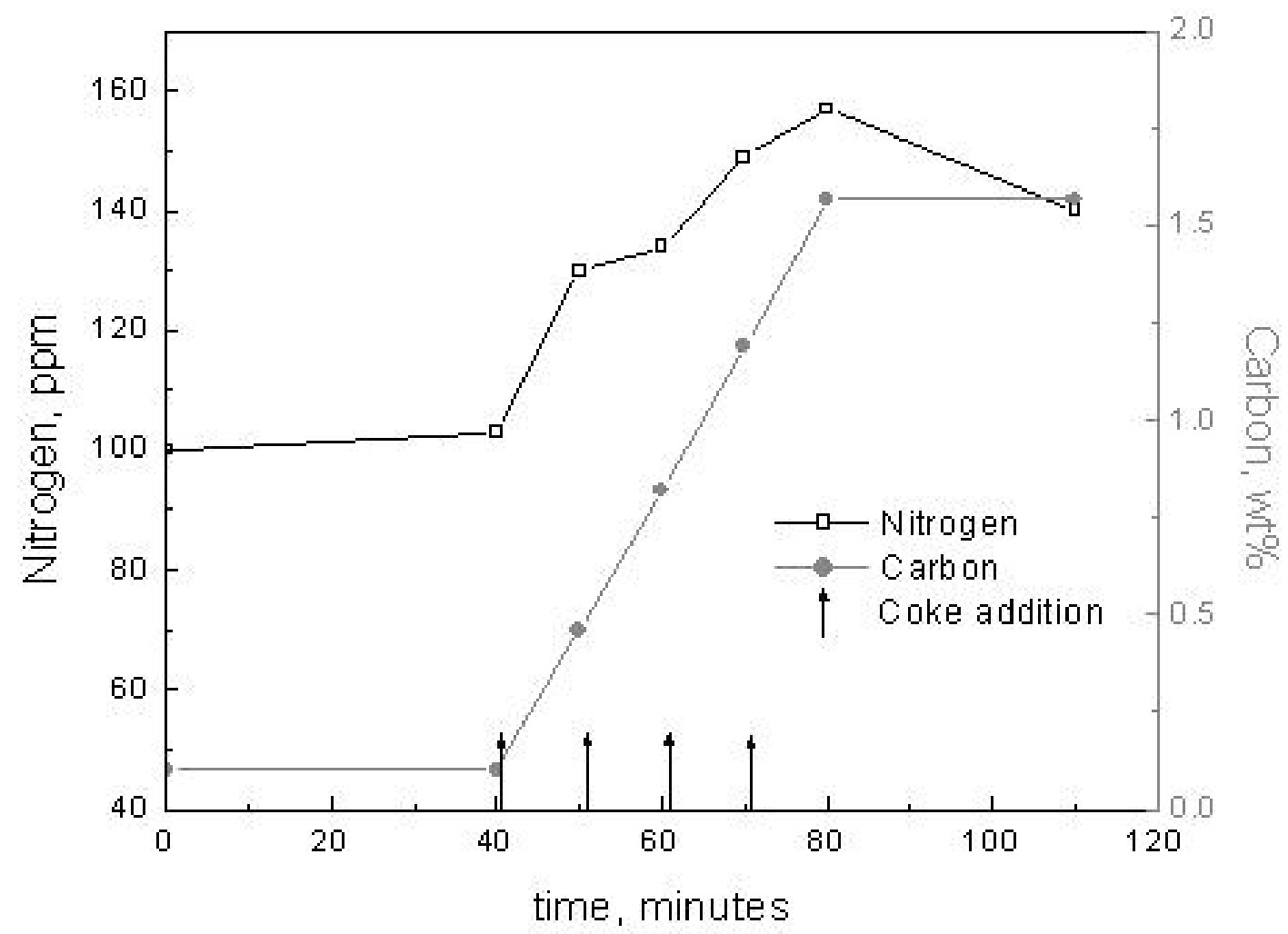

Figure 8, Effect of pitch coke addition on the nitrogen and carbon content, Trial 3

\section{Discussion}

It can be seen from Figure 8 and Figure 9 that some of the dissolve nitrogen was desorbing after the last coke addition. The possibility of such desorption occurring through out the experiment cannot be ruled out. In an attempt to account for the 
decrease in nitrogen content towards the end of the experiment (Trial 3 and 4), the following chemical equation must be examined

$$
2[\mathrm{~N}]=\mathrm{N}_{2}(\mathrm{~g}) \quad \text { Equation } 6
$$

The above equation represents the chemical reaction involved in nitrogen removal from liquid iron. Since the metal has very low oxygen and sulfur content, the chemical reaction is possibly not the rate controlling step. Liquid phase mass transfer of nitrogen through the liquid metal becomes primary in such a situation.

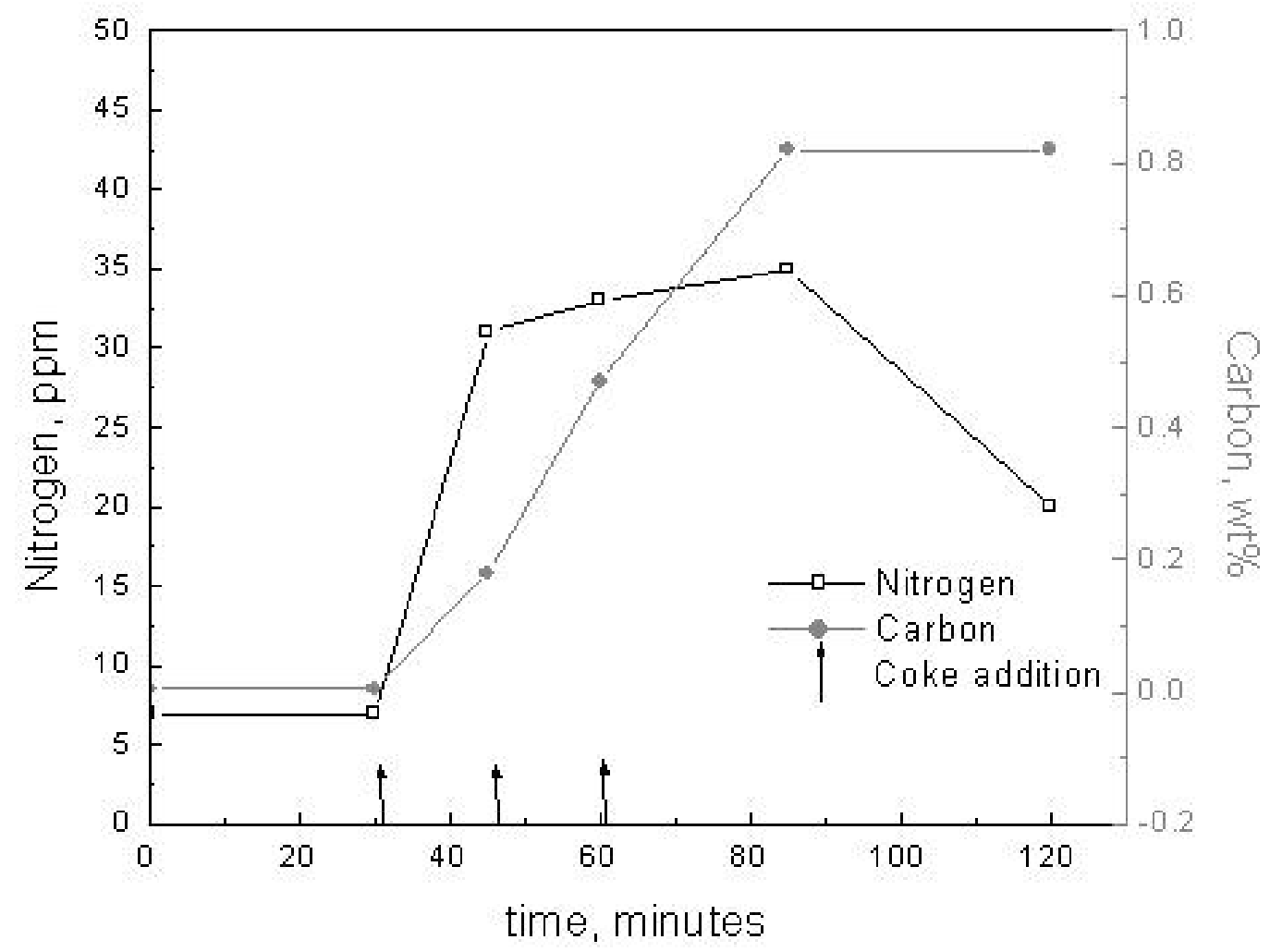

Figure 9, Effect of pitch coke addition on the nitrogen and carbon content of iron, Trial 4

The following expression represents the nitrogen content as a function of time for a liquid phase mass transfer control mechanism. 


$$
\ln \left(\frac{\left[\% N_{t}\right]-\left[\% N_{e}\right]}{\left[\% N_{o}\right]-\left[\% N_{e}\right]}\right)=-\frac{A \rho m}{W} t
$$

where $\% \mathrm{~N}_{0}$ is the initial nitrogen, $\% \mathrm{~N}_{\mathrm{t}}$ is the nitrogen at time $\mathrm{t}(\mathrm{s}), \% \mathrm{~N}_{\mathrm{e}}$ is the equilibrium nitrogen concentration (wt percent), A is the surface area of the melt $\left(\mathrm{cm}^{2}\right), p$ is the density $\left(\mathrm{g} \mathrm{cm}^{-3}\right), \mathrm{m}$ is the liquid phase mass transfer coefficient $\left(\mathrm{cm} \mathrm{s}^{-1}\right)$ and $W$ is the mass of the melt $(\mathrm{g})$.

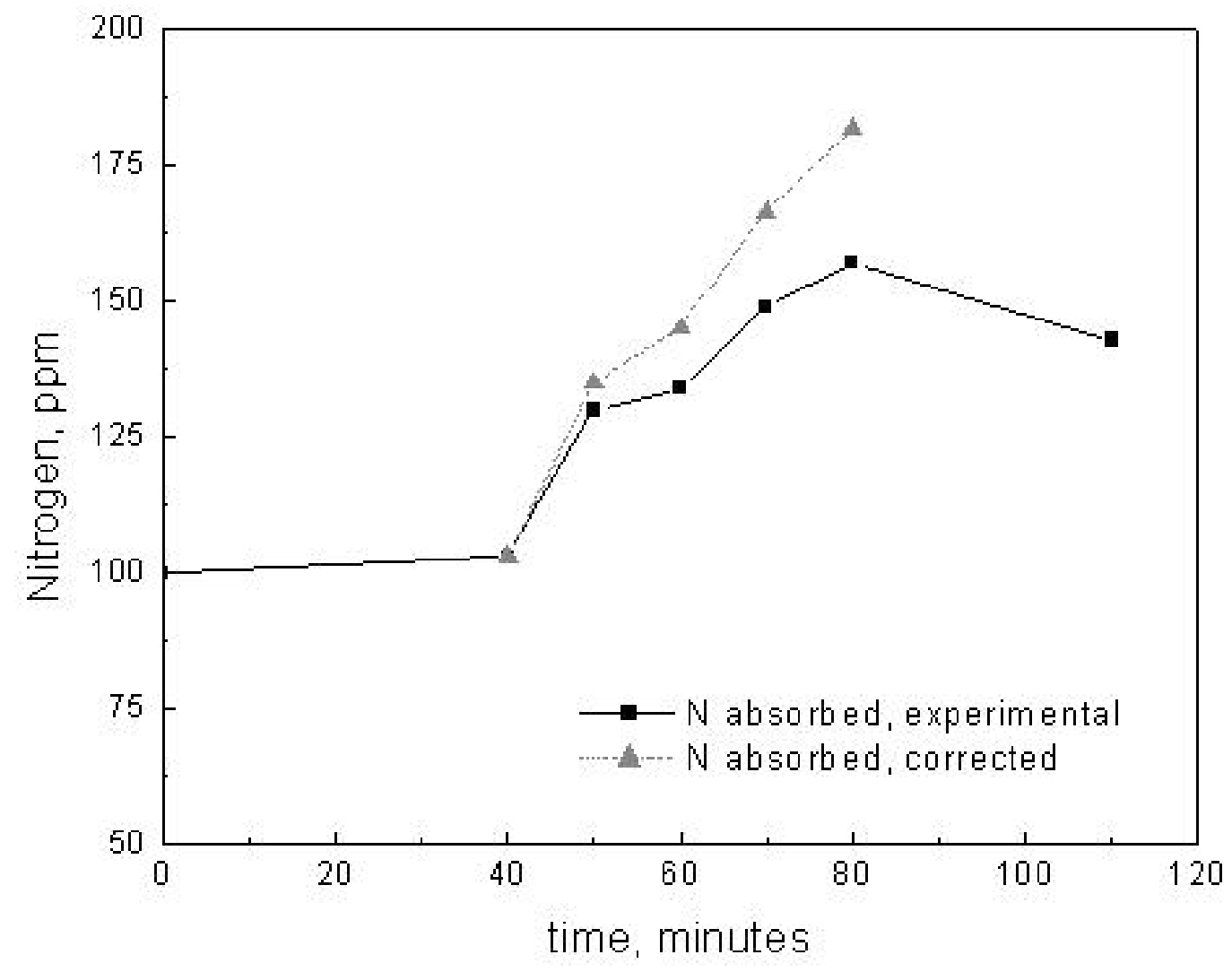

Figure 10, Nitrogen pickup corrected for the desorption, Trial 3

To estimate the extent of nitrogen desorption, calculations were performed to find the mass transfer coefficient for trials 3 and 4 . For these calculations, values from the last two data points were substituted in Equation 7. Since a constantly replenished inert atmosphere exists over the liquid metal, the equilibrium nitrogen content at the gas- 
metal surface, $\% \mathrm{~N}_{\mathrm{e}}$, is assumed to be zero. The liquid phase mass transfer coefficient for Trial 3 and Trial 4 were calculated to be $7.14 \times 10^{-4} \mathrm{~cm} / \mathrm{s}$ and $8.59 \times 10^{-4} \mathrm{~cm} / \mathrm{s}$ respectively. These values appear reasonable for given experimental conditions. For each trial, the respective mass transfer value was used to compute the amount of nitrogen which was desorbing all through the experiment. Figure 10 and Figure 11 show the nitrogen pickup corrected for desorption from the liquid metal. It can therefore be said that the actual pickup of nitrogen is much higher than what is observed experimentally.

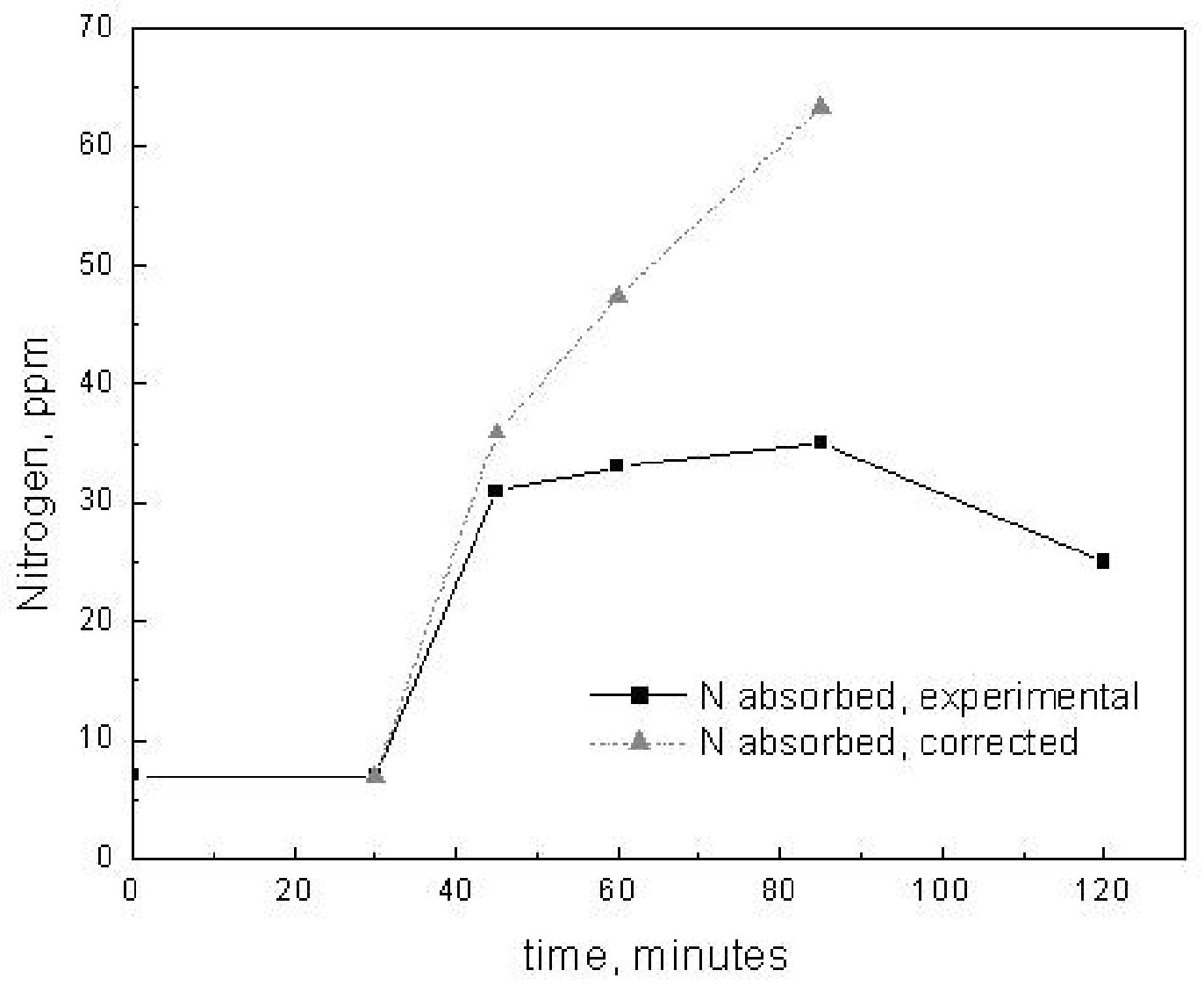

Figure 11, Nitrogen pickup corrected for the desorption, Trial 4

Presence of an inert atmosphere on top of the liquid metal may provide some driving force for nitrogen desorption. Another possibility for the low nitrogen pickup could be that not all the nitrogen reported as an impurity in the coke goes into the liquid metal. 
To elaborate, it can be said that the nitrogen in the coke may be present as a compound such as cyanides, nitrides etc. On heating the coke, the nitrogen present as volatiles releases in the form of light gases such as $\mathrm{HCN}$ and $\mathrm{NH}_{3}$. Consequently the nitrogen left in the coke is the one which exists as a non-volatile compound. During the laboratory experiments, when coke was added to the liquid metal, the volatiles escaped into the atmosphere much before the coke could reach the melt temperature. Also at lower temperatures, the rate of reaction of the gases is relatively slow so nitrogen pickup from the volatiles is negligible. This implies that the amount of nitrogen entering the melt was a fraction of the total impurity content. To confirm this hypothesis, a weighed amount of coke was heated in an argon atmosphere in a resistance furnace held at $1823 \mathrm{~K}$ for 6 hours. This heated sample along with an as received sample was sent for nitrogen chemical analysis. The results of the analysis showed that the nitrogen content decreased from $0.95 \%$ to $0.61 \%$ on heating to the steelmaking temperature.

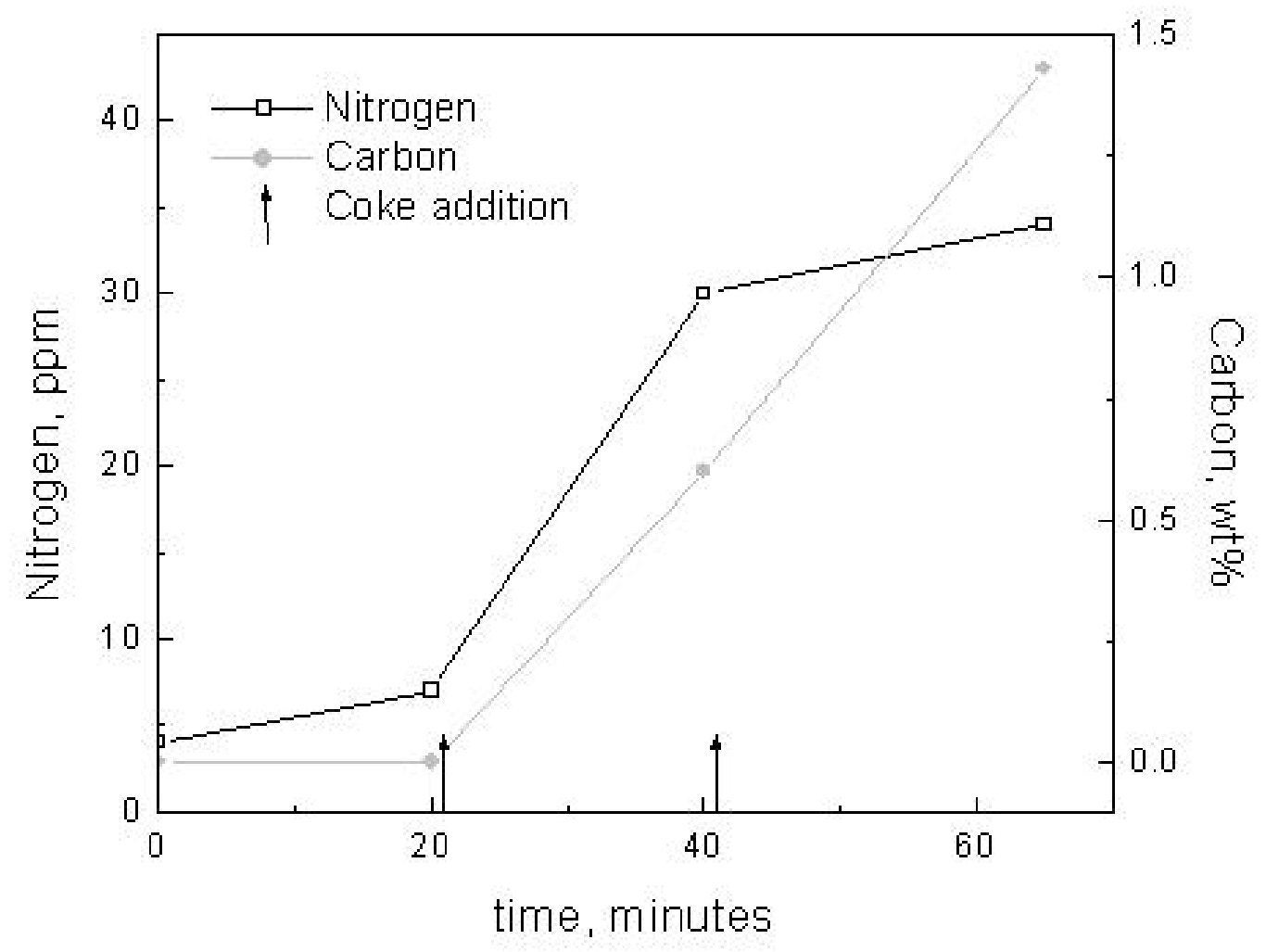

Figure 12, Effect of pitch coke (heated to $1823 \mathrm{~K}$ ) addition on the nitrogen and carbon content of iron, Trial 5 
An experiment was also performed using the coke sample which was heated to $1823 \mathrm{~K}$ to verify its effect on the nitrogen pickup. In this trial, Trial 5 , the same amount of coke was added as in the previous trial (Trial 4). The results from this experiment, given in Figure 12, illustrate that not much atmospheric nitrogen was absorbed. After the first two coke additions, the nitrogen content increased by $30 \mathrm{ppm}$. This value is consistent with the one obtained in Trial 4 (Figure 9). Such an agreement supports the argument that part of the nitrogen in the coke is present in the form of volatiles, which escape when coke reaches the steelmaking temperature. In Trial 5, such volatiles were absent in the coke sample since it was heated to $1823 \mathrm{~K}$. Similarly, in Trial 4, the nitrogen present in the volatile form escaped when the coke was added to the melt because of the increase in the coke temperature. Hence both these coke samples had the same nitrogen content soon after contact was established with the liquid metal surface. This explains the same nitrogen pickup from these two separate trials.

Table 7, Average percentage recovery of nitrogen from the coke additions

\begin{tabular}{|c|c|c|c|c|}
\hline Trial \# & 2 & 3 & 4 & 5 \\
\hline$\%$ recovery & 20.0 & 25.7 & 17.8 & 18.0 \\
\hline
\end{tabular}

Refer to Table 7 to compare the average percentage recovery of nitrogen from the coke additions in the different trials. On an average, a $20 \%$ recovery is observed. Therefore, it is reasonable to say that only a fraction of the nitrogen, which is possibly not coupled to a volatile compound, is responsible for nitrogen absorption into the liquid metal.

\section{Task III Transport of Nitrogen through Slag}

Nitrogen pickup in the metal due to the transport of atmospheric nitrogen through the slag was also studied in this research. After a number of attempts, useful data was obtained from a few experiments. The slag composition is given in Table 8 and Table 9 provides the details of the successful experiments in terms of the type of slag used and the weight of the metal and slag. In the first trial, metal samples were taken one hour 
and 24 hours after the inert gas was replaced by the nitrogen gas. In a different experiment (Trial 2) with the same slag, samples were taken 6 and 12 hours after the nitrogen flow was started. The chemical analysis results for the metal for both these trails are shown in Figure 13. From this figure it can be seen that for both the trials, a modest increase of 20 ppm nitrogen occurred over a course of 12 to 24 hours.

Table 8, Chemical composition (in wt percent) of the slag used in nitrogen transport experiments.

\begin{tabular}{|c|c|c|c|c|}
\hline Slag type & $\mathrm{Al}_{2} \mathrm{O}_{3}$ & $\mathrm{CaO}$ & $\mathrm{MgO}$ & $\mathrm{SiO}_{2}$ \\
\hline $\mathrm{MgO}$ rich & 28.30 & 50.70 & 10.12 & 10.56 \\
\hline $\mathrm{Al}_{2} \mathrm{O}_{3}$ rich & 56.99 & 26.30 & 7.86 & 8.55 \\
\hline
\end{tabular}

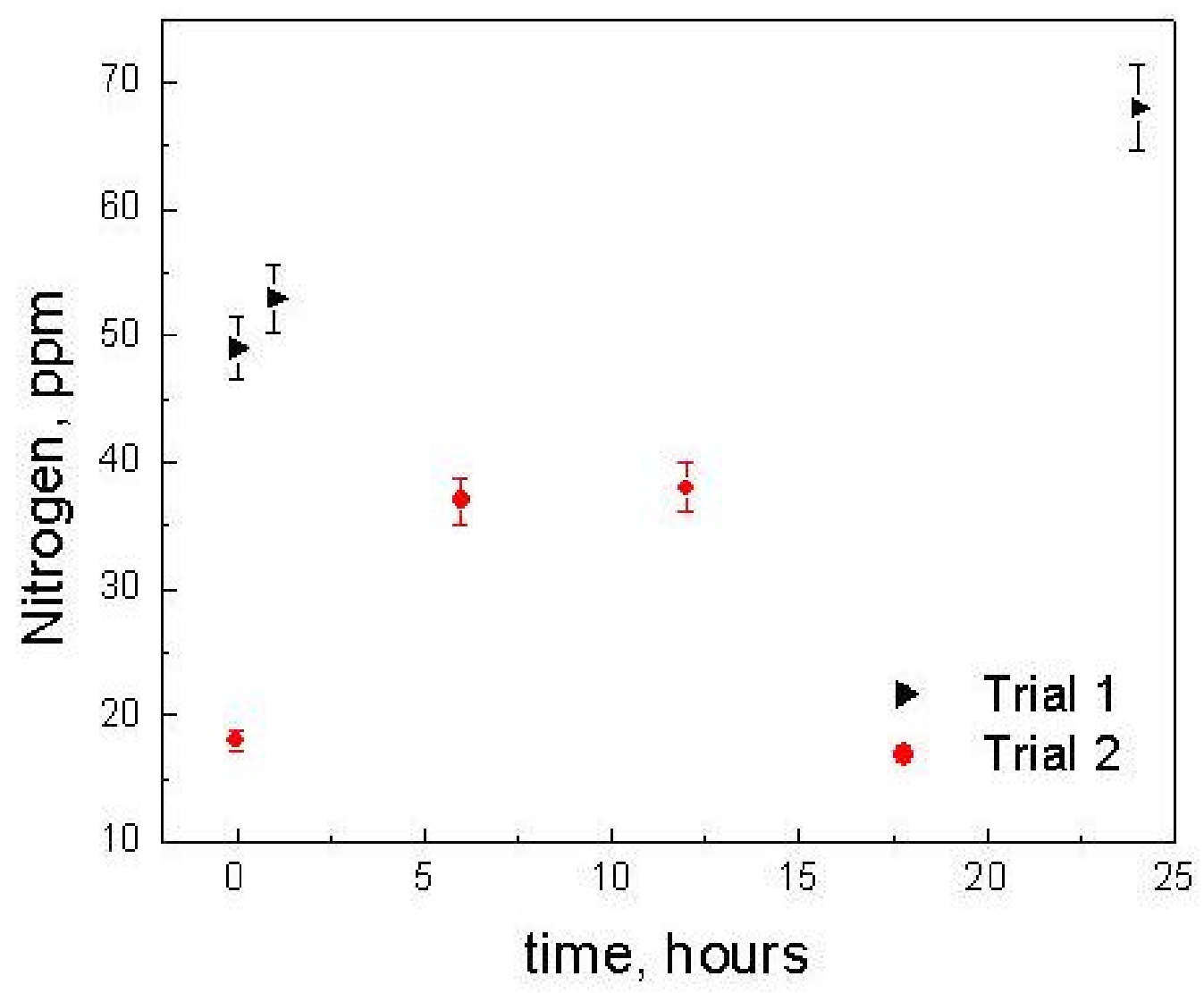

Figure 13, Nitrogen pickup through a slag layer as a function of time, Trial 1 and 2 
Table 9, Amount and type of slag-metal combination used in the transport experiments

\begin{tabular}{|c|c|c|c|c|}
\hline Experiment & Fe-Al alloy, $\mathrm{g}$ & $\% \mathrm{Al}$ & Slag, $\mathrm{g}$ & Slag type \\
\hline Trial 1 & 225 & 0.19 & 140 & MgO rich \\
\hline Trial 2 & 140 & 0.19 & 75 & $\mathrm{MgO}$ rich \\
\hline Trial 3 & 261 & 0.059 & 150 & $\mathrm{MgO}$ rich \\
\hline Trial 4 & 158 & 0.19 & 100 & $\mathrm{Al}_{2} \mathrm{O}_{3}$ rich \\
\hline
\end{tabular}

One more experiment (Trial 3) was performed with the $\mathrm{MgO}$ rich slag the results of which are shown in Figure 15. The experiment lasted for over 25 hours but a maximum pickup of only $6 \mathrm{ppm}$ was registered. Similar observation was made in an experiment performed using an $\mathrm{Al}_{2} \mathrm{O}_{3}$ rich slag. Figure 16 shows the results from this experiment, where a maximum pickup of $8 \mathrm{ppm}$ was recorded.

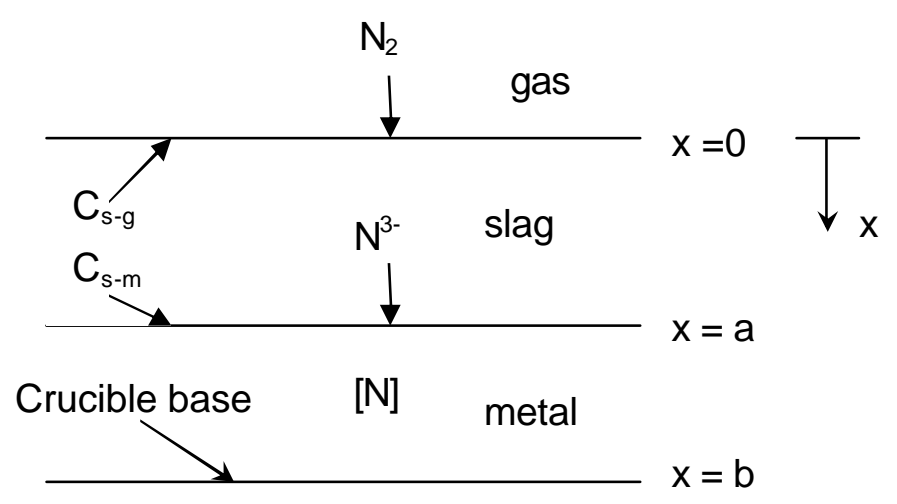

Figure 14, Schematic representation of the nitrogen diffusion through the slag layer

\section{Discussion}

Figure 14 shows a simple schematic of the gas-slag-metal system. Contrary to the common belief of slag protecting the melt from the atmospheric nitrogen, it is seen here that nitrogen gets transported through the slag layer. However, the extent of pickup from such a transport is very small and requires a longer time span for a significant absorption. In this research, calculations were performed to estimate the nitrogen pickup 
due to diffusion of nitrogen gas through the slag using the Fick's Second Law of Diffusion given as follows

$$
\frac{\partial C}{\partial t}=\frac{\partial}{\partial x}\left(D \frac{d C}{d x}\right)
$$

where $\mathrm{C}$ is the concentration, $\mathrm{x}$ is the distance, $\mathrm{t}$ is time and $\mathrm{D}$ is the diffusivity.

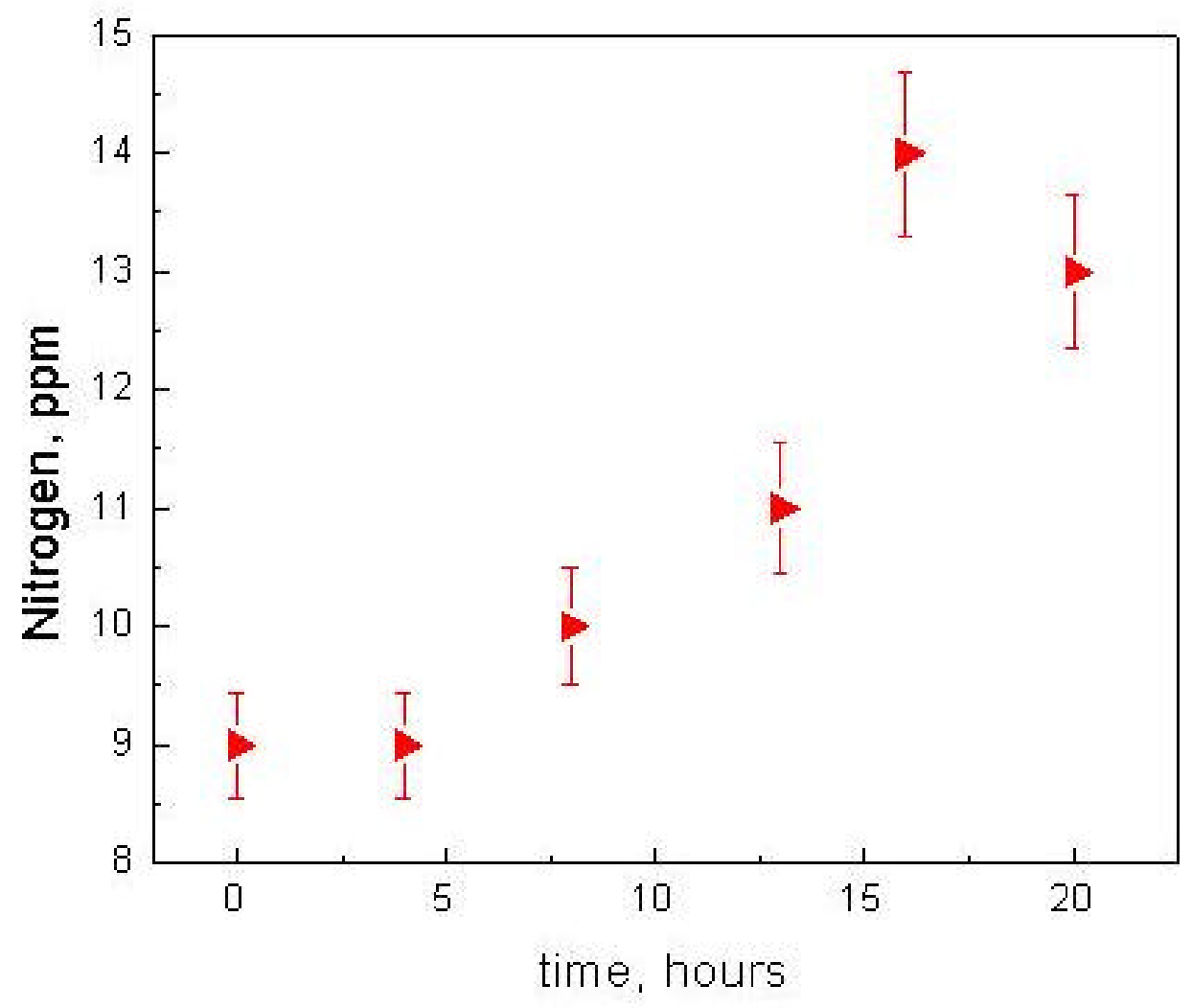

Figure 15, Nitrogen transport through MgO rich slag, Trial 3

Assuming that the diffusivity of nitrogen is independent of the concentration, for the slag and metal phase the diffusion equation simplifies to

$$
\frac{\partial C_{s}}{\partial t}=D_{s} \frac{\partial^{2} C_{s}}{\partial x^{2}} \quad \text { Equation } 9
$$




$$
\frac{\partial C_{m}}{\partial t}=D_{m} \frac{\partial^{2} C_{m}}{\partial x^{2}}
$$

where $C_{s}$ and $C_{s}$ represent the nitrogen $\left(N^{3}\right)$ concentration and $D_{s}$ and $D_{m}$ represent the nitrogen diffusivity in the slag and the metal phase respectively.

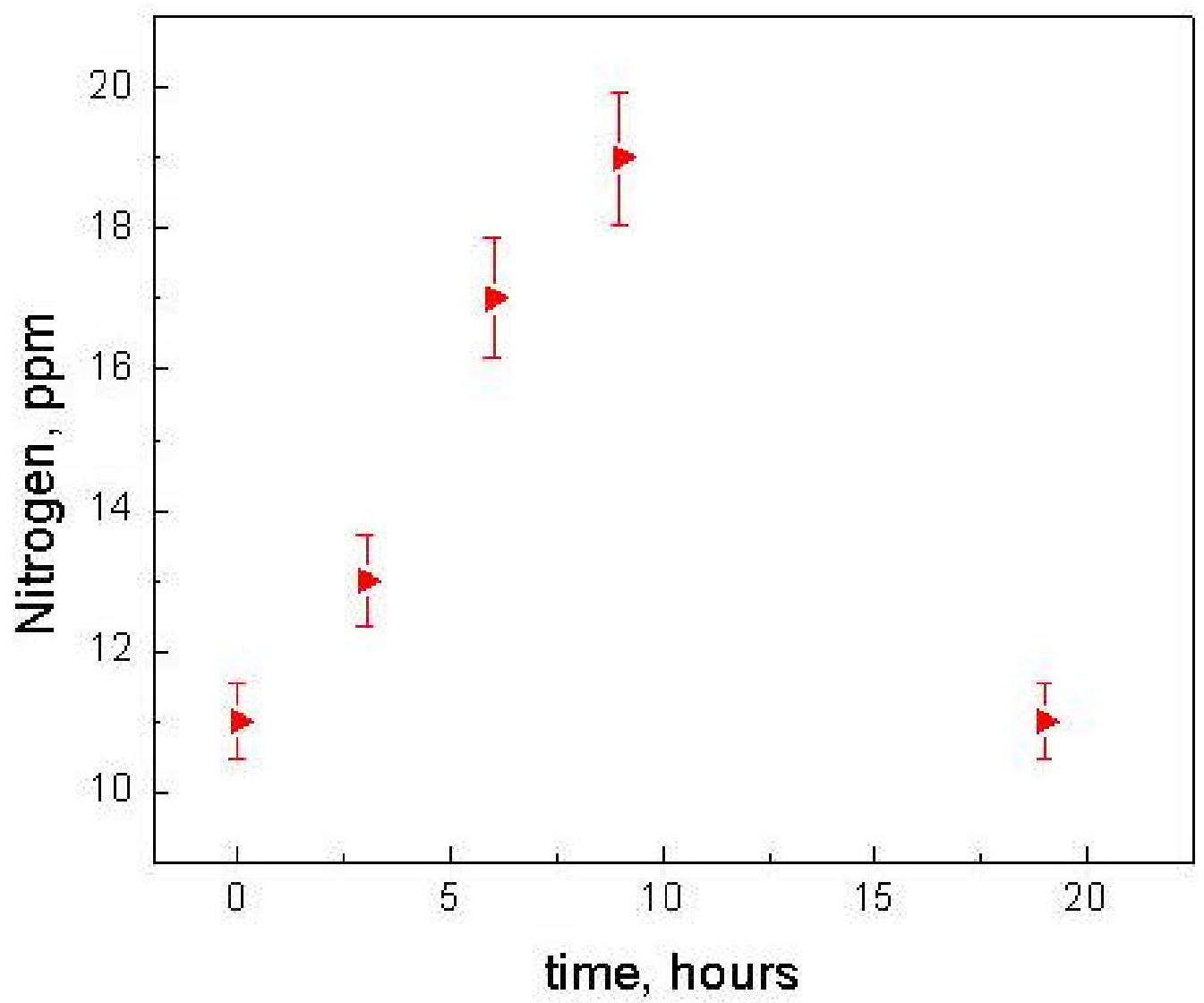

Figure 16, Nitrogen transport through an $\mathrm{Al}_{2} \mathrm{O}_{3}$ rich slag, Trial 4

Nitrogen diffusion at different locations in the slag and metal phase is calculated as a function of time by using a Finite Difference Method (FDM). In this method, Equations 9 and 10 were discretized; on such equation is given below.

$$
C_{x, j+1}=C_{x, j}+r\left(C_{x-1, j}-2 C_{x, j}+C_{x+1, j} \quad \text { Equation } 11\right.
$$


where $\mathrm{j}$ is the time $\operatorname{step}(\Delta \mathrm{t}), \mathrm{x}=$ distance step and

$$
r=\frac{D \cdot \Delta t}{\Delta x^{2}}
$$

Equation 12

The above equation is solved for both the phases using the relevant initial and boundary conditions. The partition coefficient, $\mathrm{L}_{\mathrm{N}}$, can be calculated using the nitride capacity of the slag described as follows.

$$
L_{N}=\frac{(\% N)}{[\% N]}=\frac{C_{N^{3-}}}{p_{O_{2}}^{3 / 4}} \cdot K
$$

where $\mathrm{C}_{\mathrm{N}^{3-}}$ is the nitride capacity, $\mathrm{p}_{\mathrm{O}_{2}}$ is the oxygen partial pressure (atm) and $\mathrm{K}$ is the equilibrium constant for nitrogen desorption reaction given below.

$$
\begin{array}{cc}
{[N]=\frac{1}{2} N_{2}} & \text { Equation } 14 \\
\Delta G^{o}=-3590-23.89 T\left(\mathrm{~J} \mathrm{~mol}^{-1}\right) & \text { Equation } 15
\end{array}
$$

Oxygen partial pressure is defined by the equilibrium between $\mathrm{Al}-\mathrm{Al}_{2} \mathrm{O}_{3}$

$$
\begin{array}{cc}
{[\mathrm{Al}]+\frac{3}{4} \mathrm{O}_{2}=\frac{1}{2}\left(\mathrm{Al}_{2} \mathrm{O}_{3}\right)} & \text { Equation } 16 \\
\Delta G^{o}=-778422+189.99 T\left(\mathrm{~J} \mathrm{~mol}^{-1}\right) & \text { Equation } 17
\end{array}
$$




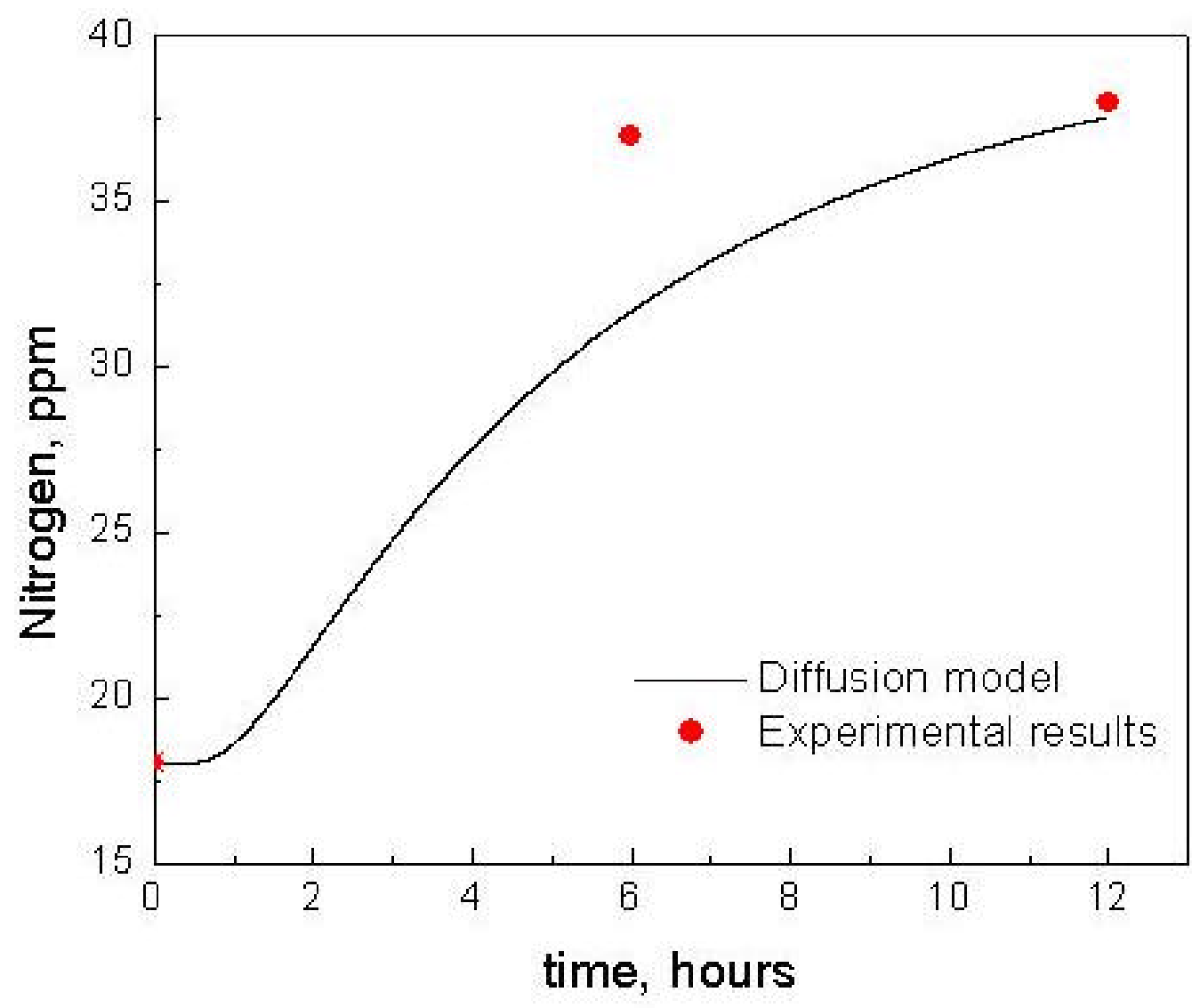

Figure 17, Nitrogen transport through slag layer, calculated and experimental, Trial 2

The activities of alumina in the slag, $\mathrm{a}_{\mathrm{Al}_{2} \mathrm{O}_{3}}$, were calculated using the FactSage Thermochemical Software and Database and were found to be $6.745 \times 10^{-4}$ and $7.125 \mathrm{x}$ $10^{-2}$ for $\mathrm{MgO}$ rich and $\mathrm{Al}_{2} \mathrm{O}_{3}$ rich slags respectively. These values were subsequently used to estimate the partial pressure of oxygen. The diffusion coefficient of nitrogen in the slags depends on its composition and is found to lie within the same order of magnitude for typical ladle slags. For a slag having a composition similar to the one used in the present experiments, the $D$ value has been reported as $5 \times 10^{-10} \mathrm{~m}^{2} \mathrm{~s}^{-1}[4$, 5] and is used for the present calculations. As reported in the literature [6], the diffusion coefficient of nitrogen in the metal phase is taken as $1.1 \times 10^{-8} \mathrm{~m}^{2} \mathrm{~s}^{-1}$. 


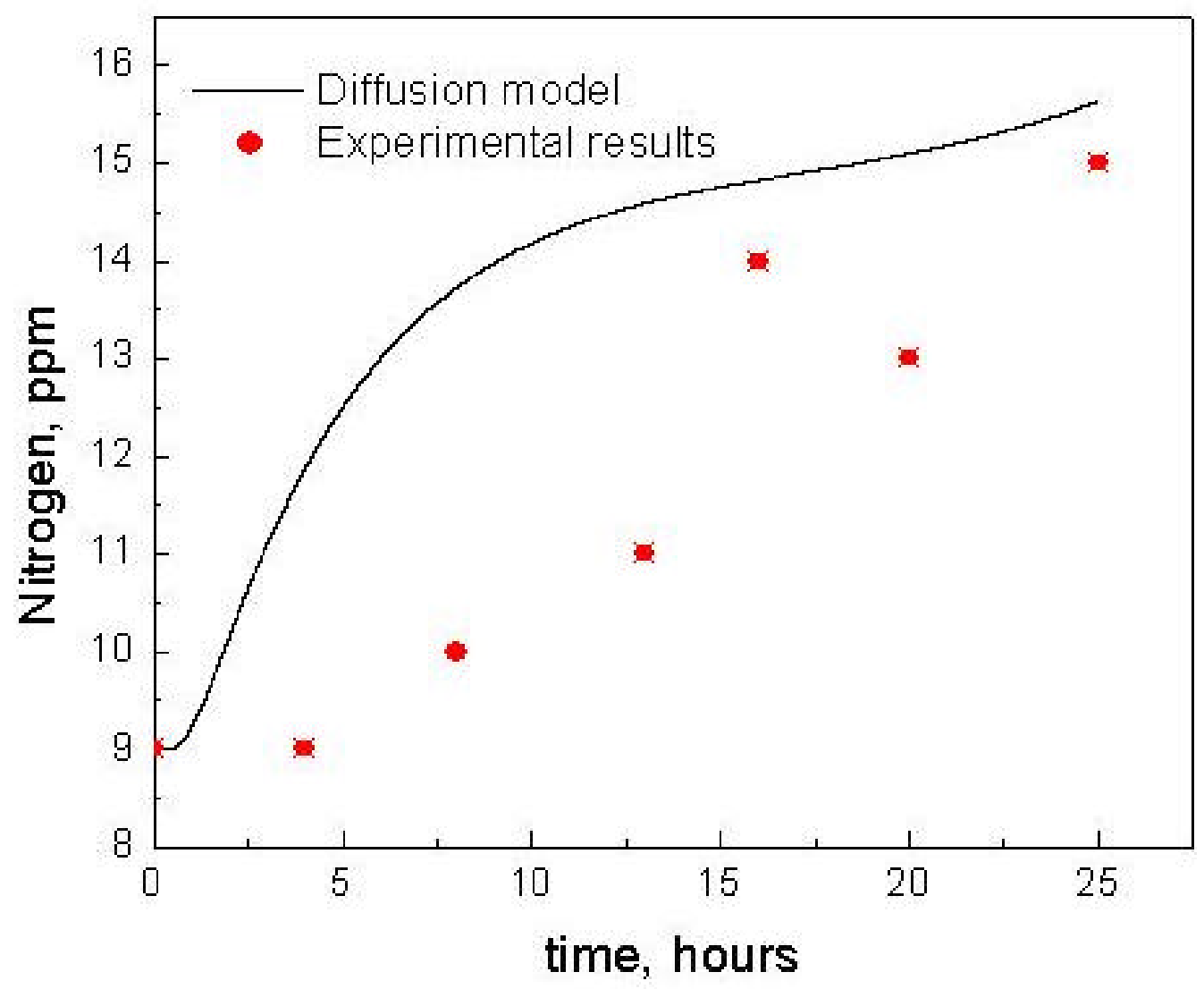

Figure 18, Nitrogen transport through slag layer, calculated and experimental, Trial 3

Using the previous set of equations to solve for the diffusion of nitrogen through the slag layer, the nitrogen content of the metal was calculated and is shown in Figure 17, Figure 18 and Figure 19. The diffusion model seems to predict the nitrogen transport to a reasonable extent. The initial lag in the nitrogen pickup, depicted by the flattened tail in the diffusion model, is due to time taken for the nitrogen to diffuse through the slag layer before it can enter the liquid metal. After this initial phase when some nitrogen has been transported from the atmosphere into the slag phase, the nitrogen content of the liquid metal starts increasing. The agreement between the experimental and the model predictions is considered excellent considering the uncertainties in the nitride capacity, oxygen potential and nitrogen diffusivity values. It can be concluded, therefore, that atmospheric nitrogen through the slag layer is very slow and possibly diffusion controlled. 


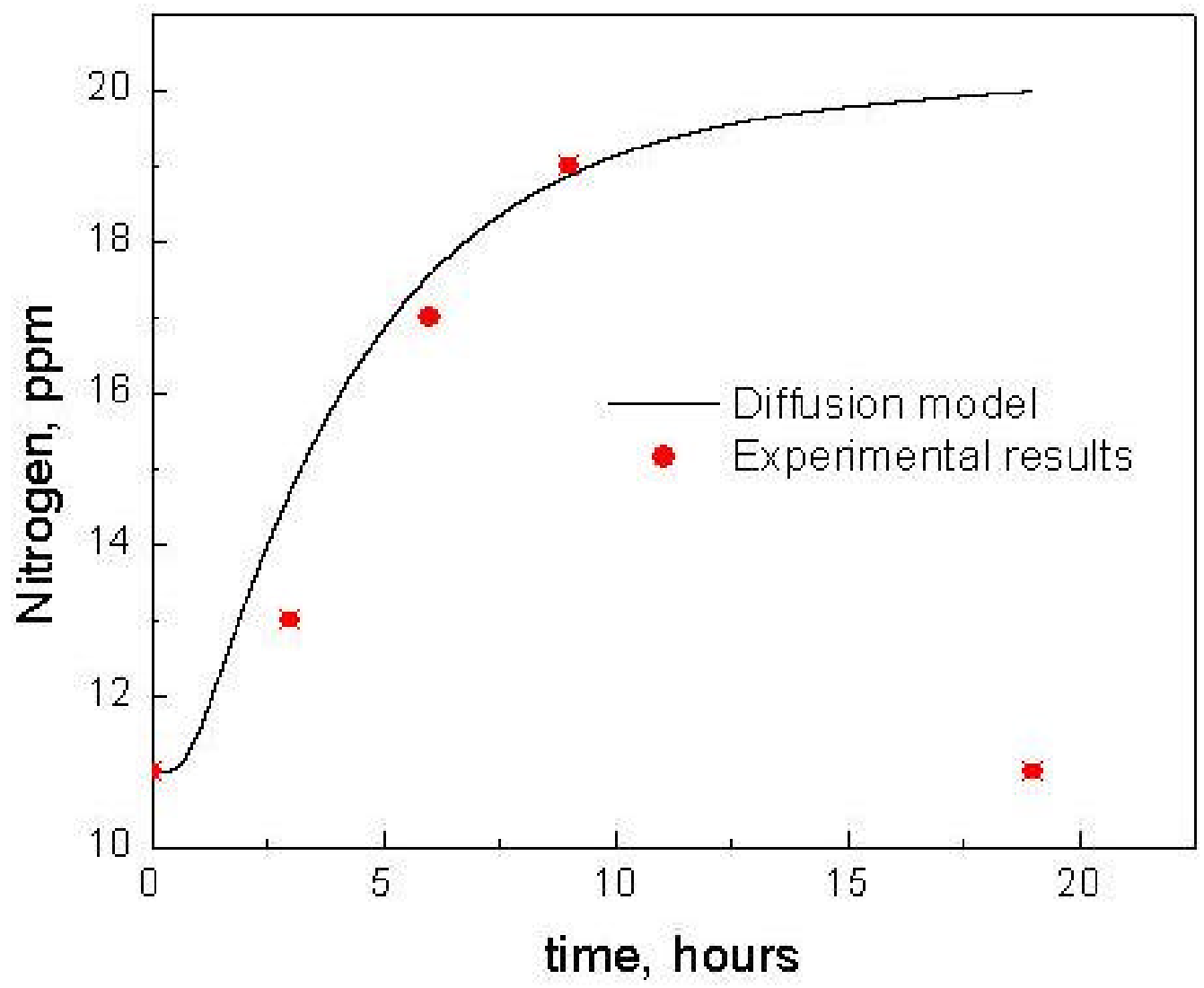

Figure 19, Nitrogen transport through slag layer, calculated and experimental, Trial 4

\section{Task IV Model for ladle Processing}

\section{(a) Nitrogen and Hydrogen Pickup during Tapping of Liquid Steel}

Nitrogen and hydrogen absorbed from the entrained air bubbles were calculated using the mixed control models developed for each case. The effects of various variables were also explored. To incrementally solve for the nitrogen and hydrogen pickup, computer codes were written in C. In these computer programs, the various variables used in the rate equation were defined and calculations were performed for a 1 tonne per second tapping rate for a 100 tonne tap. For most of the calculations, the starting nitrogen and hydrogen (before tap) were reasonably assumed to be $30 \mathrm{ppm}$ and 
$3 \mathrm{ppm}$ respectively and the liquid metal temperature was taken as $1873 \mathrm{~K}$. The liquid phase mass transfer coefficient used for hydrogen pickup calculations has been taken from the work of Fruehan and Martonik [7] and is of the value $9.7 \times 10^{-2} \mathrm{~cm} \mathrm{~s}^{-1}$. The interfacial area between the air-liquid metal was calculated for a 5 degree ladle slice hence the total area was 72 times the calculated value. For all the computations, the carbon content of the metal was assumed to be $0.02 \mathrm{wt} \%$.

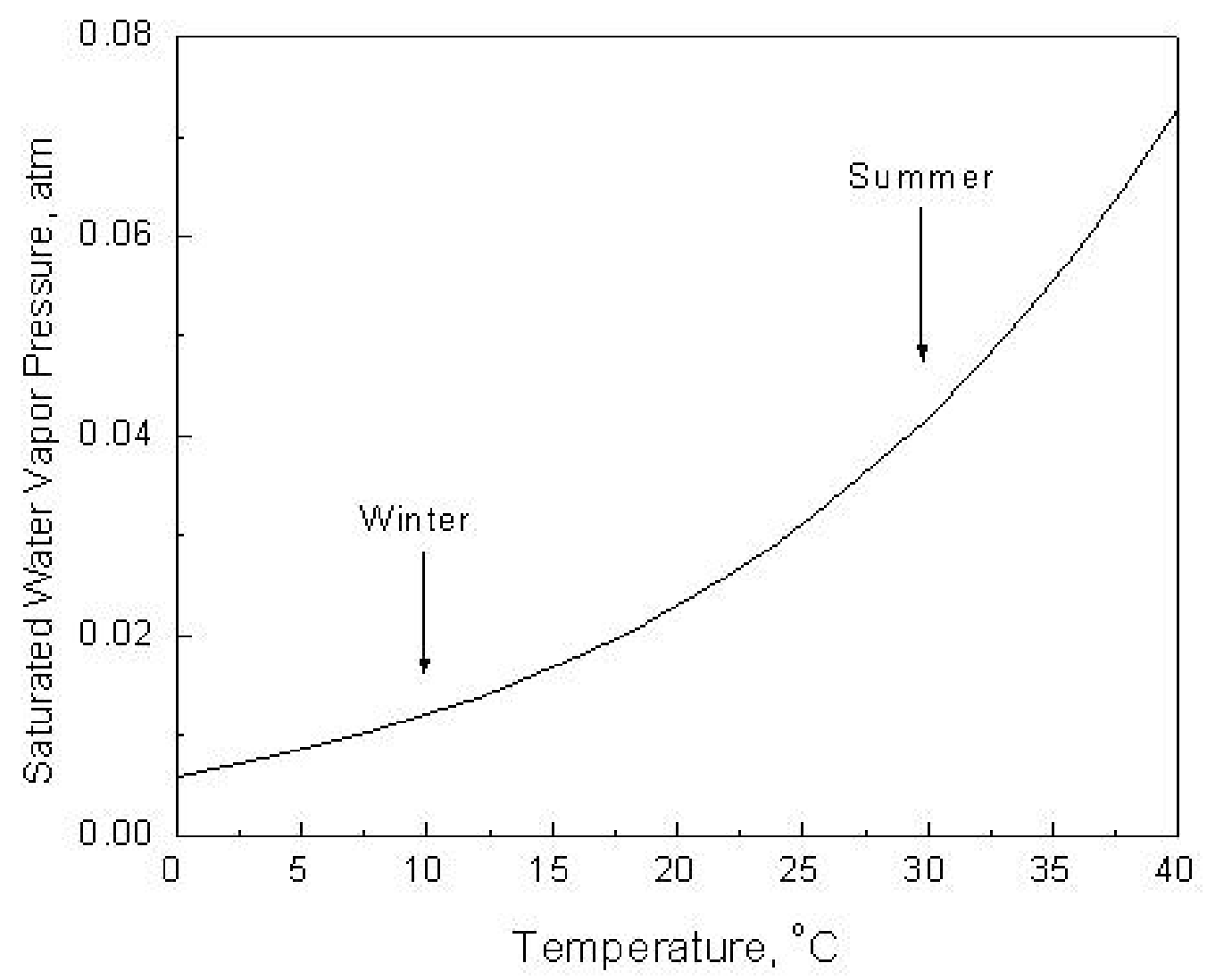

Figure 20, Water vapor partial pressure as a function of temperature [8]

\section{Effect of Water Vapor Pressure on the Hydrogen Pickup}

The extent of hydrogen pickup strongly depends on the partial pressure of water vapor in the atmosphere. Figure 20 shows the saturated vapor pressure of water in the air at different temperatures. It is noteworthy that the amount of moisture in the air will depend on the temperature and the relative humidity. Hence, for any given temperature, 
the water vapor partial pressure will be the product of the relative humidity and the saturated vapor pressure. For instance, on a typical summer day when the relative humidity is as high as $80 \%$, the water vapor pressure will be around 0.04 atmospheres while on a winter day for a relative humidity of $30 \%$ the vapor pressure is less than 0.01 atmospheres. Nonetheless high moisture content in the ambient atmosphere is a favorable condition for a higher hydrogen pickup. The effect of different partial pressures of water vapor was therefore investigated.

\section{Effect of the Oxygen Content of the Metal, $h_{0}$}

Since oxygen acts as a surface active element and retards the rate of nitrogen absorption in liquid iron, nitrogen pickup was estimated for different oxygen contents. Figure 21 shows the nitrogen pickup for different values of the oxygen concentration. The starting nitrogen content was assumed to be $30 \mathrm{ppm}$ and the sulfur content was taken as 0.005 wt\%. Calculations were performed for two different values of liquid phase mass transfer coefficients $\left(m_{L}\right)$. Increasing the value of $m_{L}$ facilitates the intermixing of the liquid metal and hence more nitrogen is picked up which is also seen in the following figure. It should be noted that more nitrogen is absorbed for a lower oxygen content in the metal namely for a deoxidized steel. It is also important to note that it is not the oxygen in solution in the bulk metal but the oxygen at the bubble surface which determines the kinetics of the nitrogen reaction. Presence of low oxygen content facilitates a high hydrogen pickup in accordance with reaction given below

$$
\mathrm{H}_{2} \mathrm{O}=2[\mathrm{H}]+[\mathrm{O}] \quad \text { Equation } 18
$$

Also, oxygen acts as a surface active element and retards the rate of hydrogen absorption in liquid iron, therefore hydrogen pickup was estimated for different oxygen activities. Once again it is important to note that it is the oxygen activity at the bubble surface which influences the hydrogen absorption reaction. Figure 22 shows the hydrogen pickup for different values of the oxygen concentration and a constant sulfur content of 0.02 wt\%. Calculations were performed for different values of $\mathrm{H}_{2} \mathrm{O}$ partial pressures. It was found that increasing the value of $\mathrm{p}_{\mathrm{H}_{2} \mathrm{O}}$ helped in a higher hydrogen 
pick up. Also, more hydrogen was absorbed for a lower oxygen content in the metal namely for a de-oxidized steel.

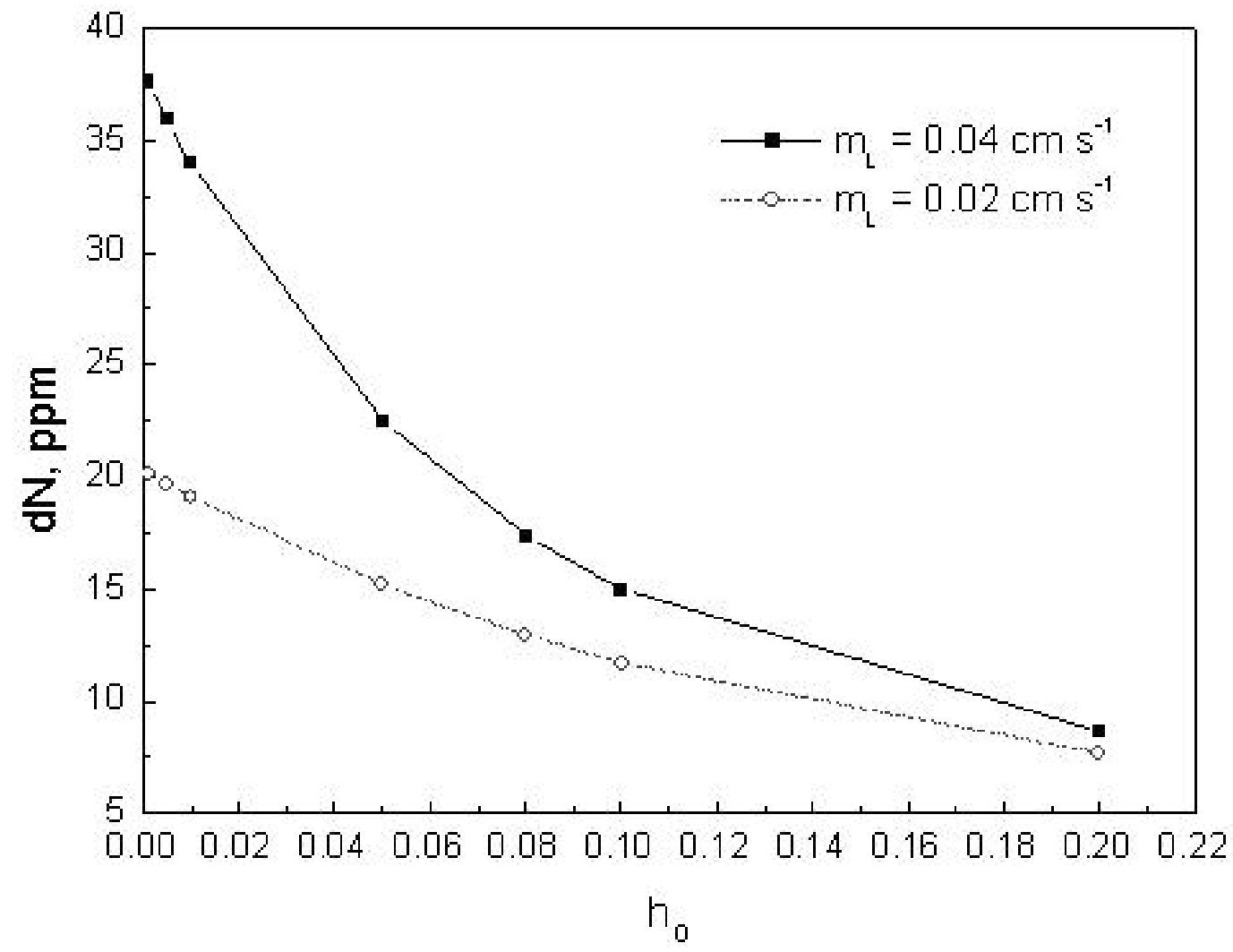

Figure 21, Nitrogen pickup for different oxygen activity at the bubble surface, $\mathrm{N}=30 \mathrm{ppm}$, $\% \mathrm{~S}=0.005 \%$

The rate of nitrogen and hydrogen pickup is controlled by the liquid phase mass transfer for lower values and by chemical kinetics for higher values of the oxygen concentration. For the intermediate concentration range, the rate is controlled by both of these resistances in series. The entrained air bubble has 0.21 moles of oxygen balance being nitrogen and water vapor. Depending upon the type of tapping, this oxygen would react with the liquid iron or the alloy elements in the melt. Consequently the oxygen concentration at the bubble-liquid metal interface is what affects the reaction kinetics. 


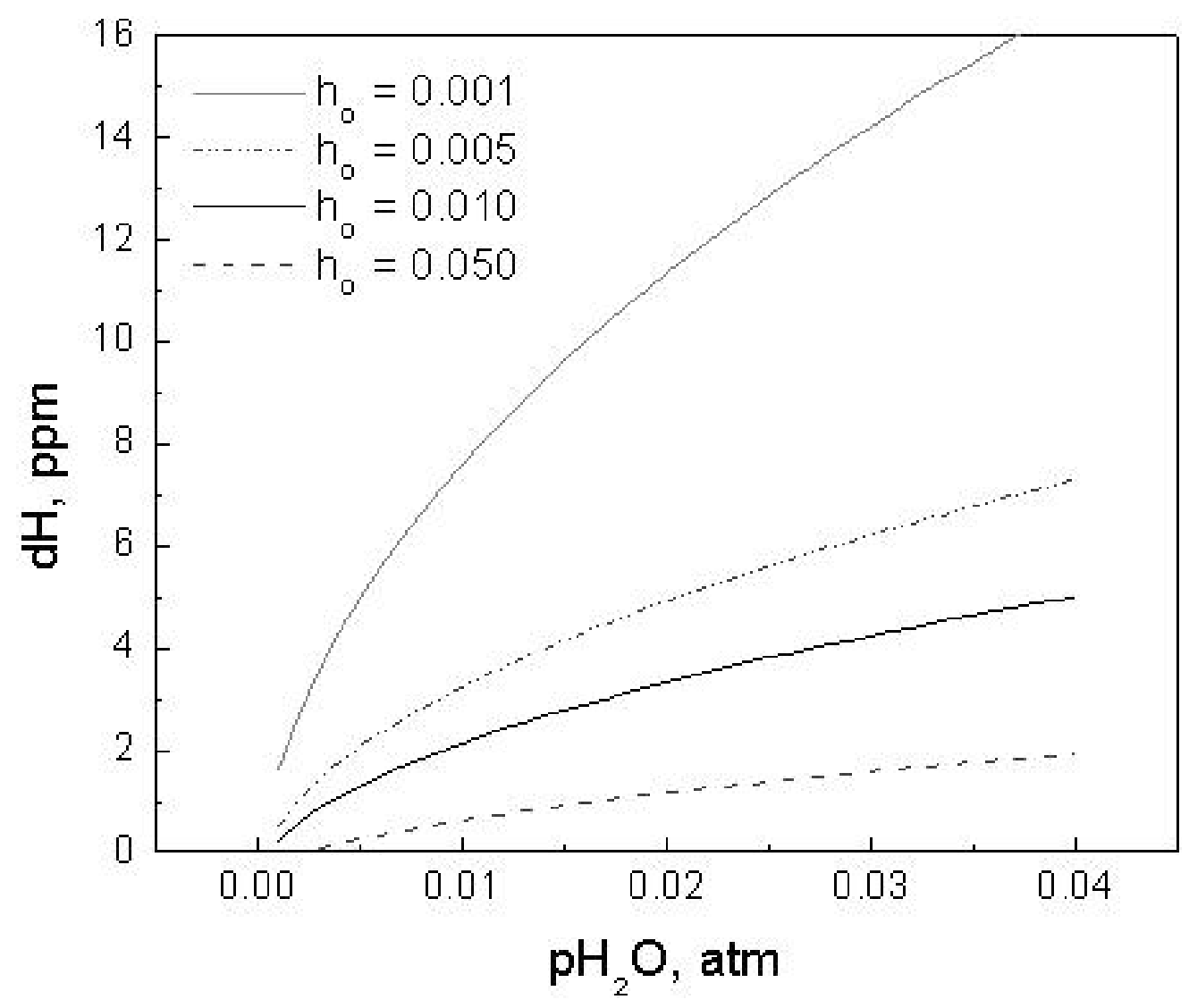

Figure 22, Hydrogen pickup for different oxygen activity at the bubble surface, $\mathrm{H}=3 \mathrm{ppm}$, $\% \mathrm{~S}=0.02, \mathrm{~m}_{\mathrm{L}}=0.097 \mathrm{~cm} \mathrm{~s}^{-1}$

\section{Effect of the Sulfur Content of the Metal, $\mathbf{h}_{\mathrm{s}}$}

Like oxygen, sulfur also retards the rate of nitrogen and water dissociation reaction with the liquid iron because of its surface active property. Figure 23 shows the effect of different sulfur concentrations on the nitrogen pickup by the liquid metal for an oxygen activity of 0.001 . For the case where the mass transfer coefficient in the liquid phase is $0.04 \mathrm{~cm} \mathrm{~s}^{-1}$ the degree of nitrogen pickup is high. In the other case, where $\mathrm{m}_{L}=$ $0.2 \mathrm{~cm} \mathrm{~s}^{-1}$, the nitrogen pickup is lower. Nevertheless, the amount of nitrogen absorption decreases as the sulfur concentration is increased. 


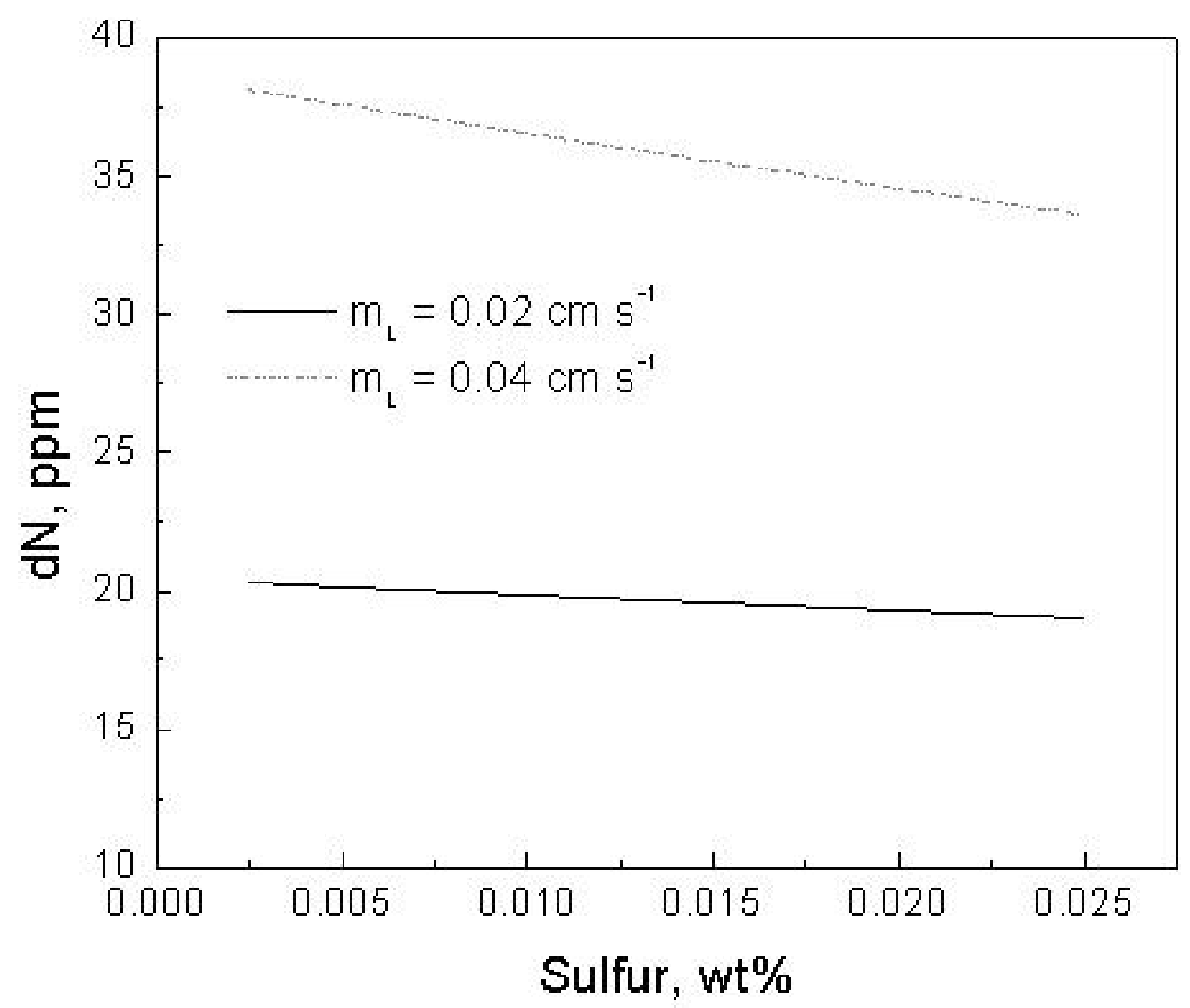

Figure 23, Nitrogen pickup for different sulfur content, $\mathrm{N}_{\mathrm{i}}=30 \mathrm{ppm}, \mathrm{h}_{\mathrm{O}}=0.001$

Similar to its effect on the nitrogen absorption, sulfur also retards the rate of $\mathrm{H}_{2} \mathrm{O}$ reaction with the liquid iron because of its surface active property. Its pronounced effect on the hydrogen absorption can be seen in highly deoxidized steel. When the oxygen content of the liquid metal is very low, it is susceptible to significant hydrogen pickup, however, presence of a high sulfur level prevents hydrogen absorption due to its surface active property. This is illustrated in Figure 24 below. 


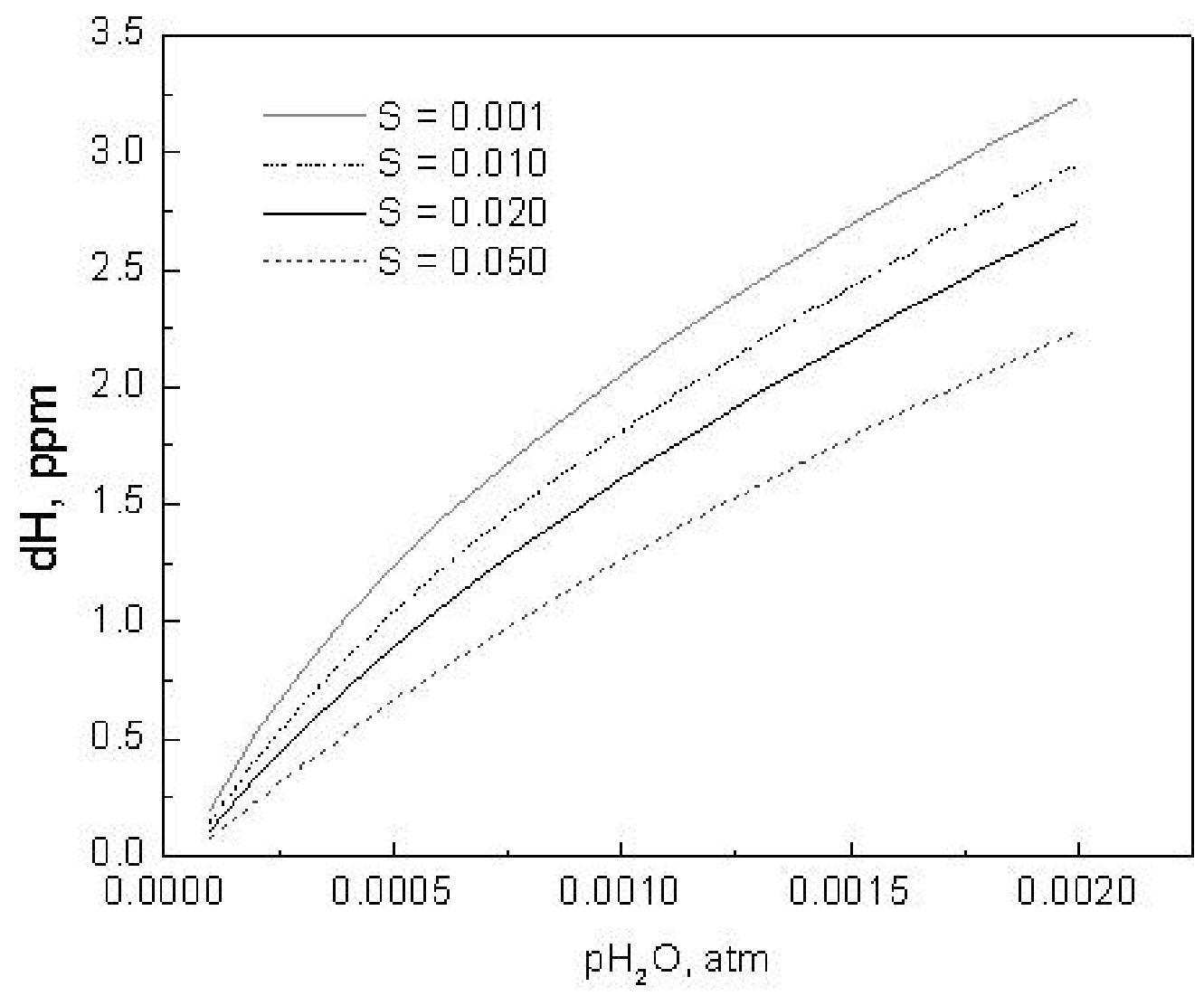

Figure 24, Hydrogen pickup for different sulfur concentrations at the bubble surface, $H_{i}=3 p p m, h_{O}=0.001, m_{L}=0.097 \mathrm{~cm} \mathrm{~s}^{-1}$

\section{Effect of Different Liquid Phase Mass Transfer Coefficients, $\mathrm{m}_{\mathrm{L}}$}

Since it is difficult to obtain accurate values of mass transfer coefficients in the liquid phase, nitrogen pickup is estimated for different $m_{\llcorner}$values for the sake of comparison. Earlier research [9] reports indicate that $m_{\llcorner}$lies between 0.01 and $0.05 \mathrm{~cm} \mathrm{~s}^{-1}$ so there values were used for comparison. The sulfur content of the metal is assumed to be $0.005 \mathrm{wt} \%$ and the starting nitrogen content of $30 \mathrm{ppm}$ is considered. Nitrogen pickup is calculated for the three oxygen concentrations. It is seen from Figure 25 that the nitrogen pickup increases with the increasing values of $m_{L}$. Higher pickup is also found for a decreasing oxygen content of the metal. 


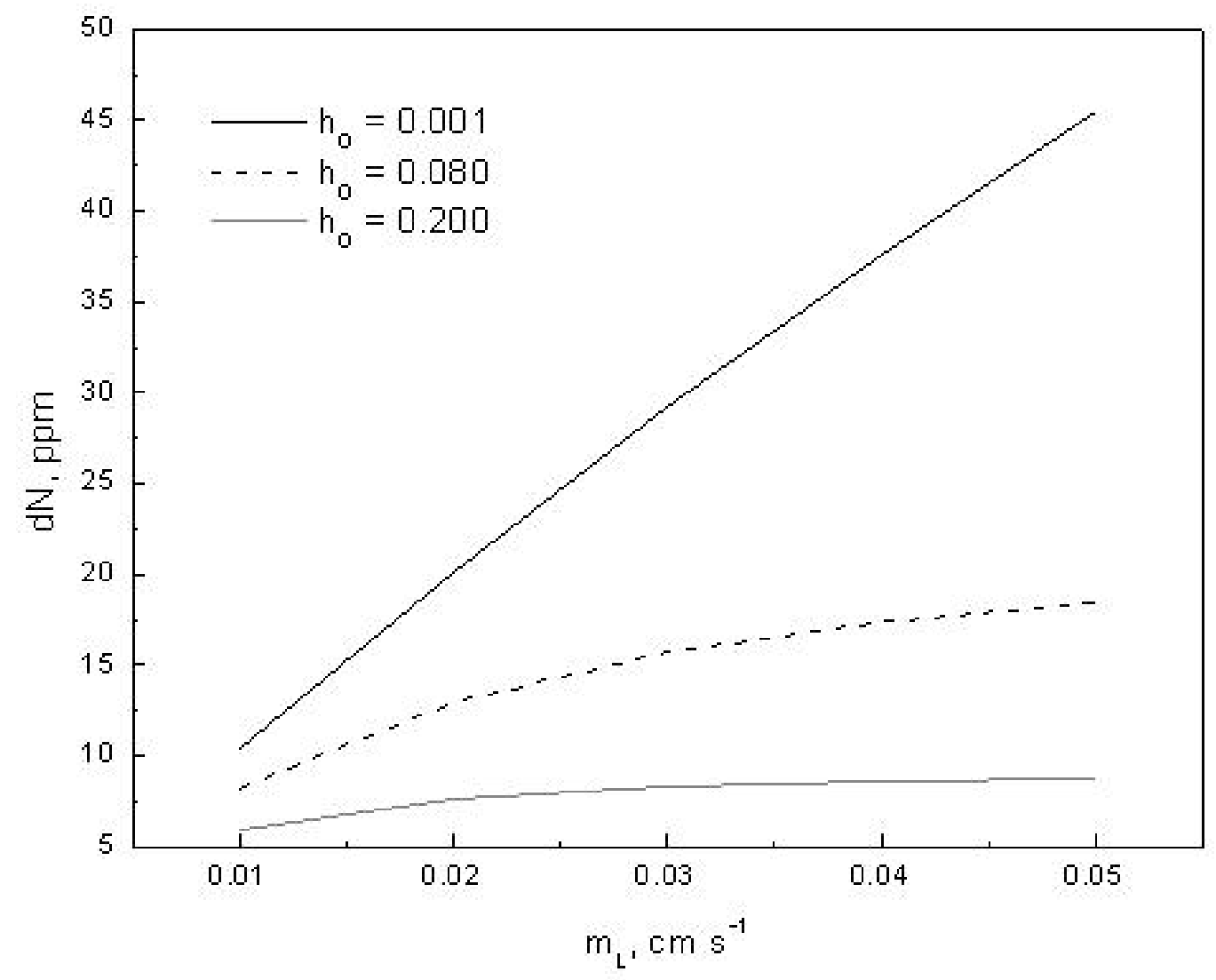

Figure 25, Nitrogen pickup for different liquid phase mass transfer coefficients, $\mathrm{Ni}=30$ ppm, \%S $=0.005$

Since it is difficult to obtain accurate values of mass transfer coefficients in the liquid phase, hydrogen pickup is estimated for different $m$ values for the sake of comparison. From the earlier research it is known that $\mathrm{m}$ lies around $0.1 \mathrm{~cm} \mathrm{~s}^{-1}$ [7] hence values in the same vicinity are used for comparison. Hydrogen pickup is calculated for the increasing pressure of $\mathrm{H}_{2} \mathrm{O}$ for liquid steel before it is fully deoxidized and is shown in Figure 26. The oxygen activity is around 0.05 and the sulfur content is assumed to be $0.02 \mathrm{wt} \%$. A higher pickup is also found with increasing partial pressure of water vapor. 


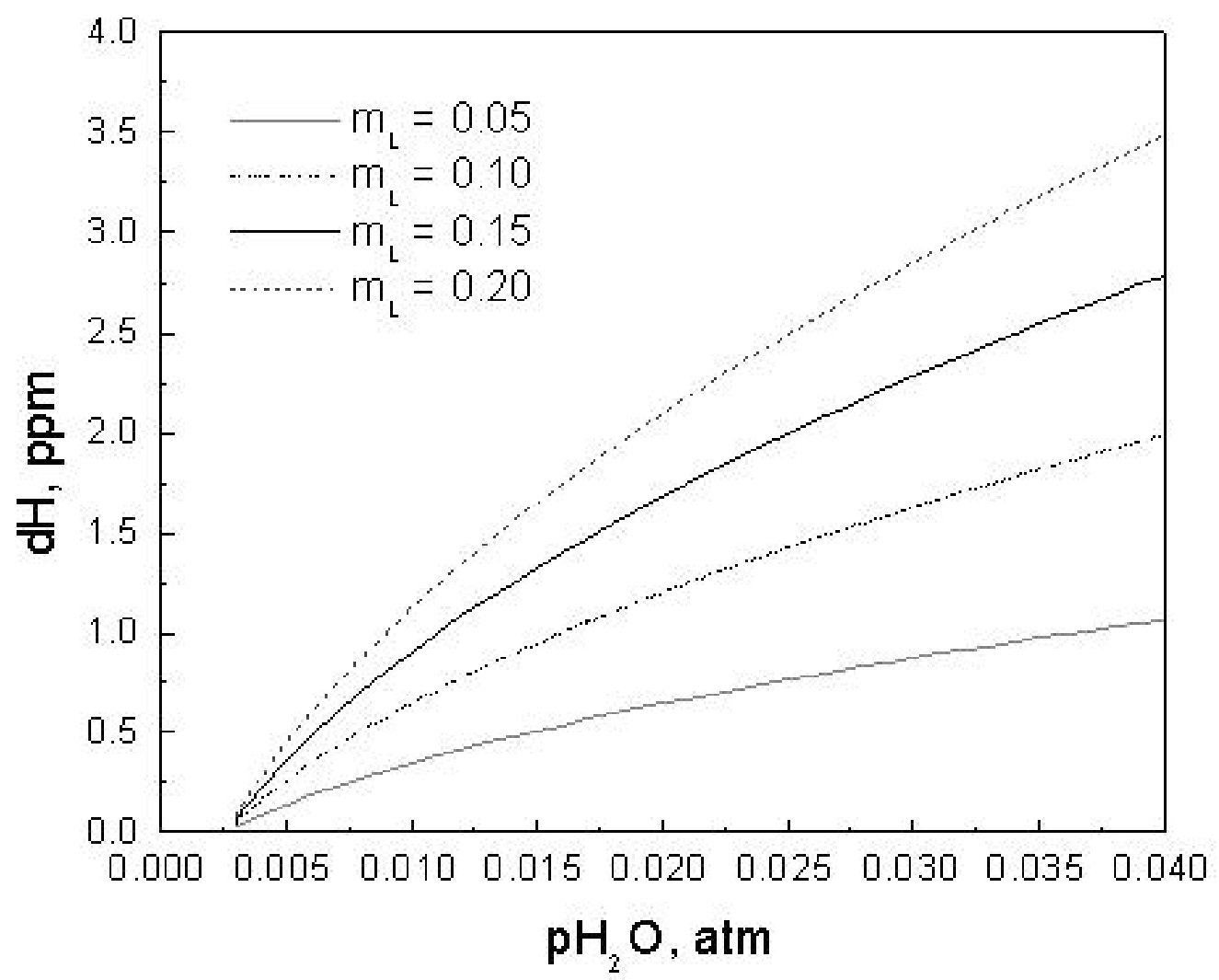

Figure 26, Hydrogen pickup for different mass transfer coefficients, $\mathrm{H}_{\mathrm{i}}=3 \mathrm{ppm}, \% \mathrm{~S}=0.02$, $h_{\mathrm{O}}=0.05$

\section{Effect of Different Tap Nitrogen and Hydrogen Contents}

Finally the effect of a different starting nitrogen content (nitrogen content before tap) was investigated. It was found that for a lower initial nitrogen concentration, the pickup is slightly greater. For deoxidized steel, with a high liquid phase mass transfer coefficient, the pickup is found to depend more on the initial nitrogen content. The effect of a different starting hydrogen content (hydrogen before tap) is also investigated. It is found that the extent of hydrogen pickup decreases slightly with an increase in starting hydrogen content. 


\section{(b) Nitrogen Pickup by Argon Stirring}

Nitrogen absorption into liquid iron was calculated using a mixed control kinetic model which has been described in sufficient detail in previous reports. Since the diffusivity of nitrogen in air is high, the rate of mass transfer in the gas phase was assumed to be fast. The absorption reaction was controlled by chemical kinetic control and liquid phase mass transfer in series. The reaction area, given by the spout eye area, was computed using the correlation developed by Subagyo et al. [10] which is given below

$$
\frac{A_{e s}}{(H+h)^{2}}=(0.02 \pm 0.002)\left(\frac{Q^{2}}{g H^{5}}\right)^{0.375 \pm 0.0136}
$$

where:
$A_{e s} \quad$ is the spout eye area $\left(\mathrm{m}^{2}\right)$
$\mathrm{h} \quad$ is the metal height $(\mathrm{m})$
$\mathrm{H} \quad$ is the slag thickness $(\mathrm{m})$
Q is the gas flow rate at nozzle exit $\left(\mathrm{m}^{3} \mathrm{~s}^{-1}\right)$
$\mathrm{g}$ is the acceleration due to gravity $\left(\mathrm{m} \mathrm{s}^{-1}\right)$

The spout eye radius was also calculated using the area calculations. In calculating the mass transfer coefficient using Equation (20), the diffusivity of nitrogen in liquid iron was taken as $1.1 \times 10^{-8} \mathrm{~m}^{2} \mathrm{~s}^{-1}[6]$.

$$
m_{L}=2\left(\frac{D \cdot u}{\pi \cdot L}\right)^{1 / 2}
$$

Equation 20

where $D$ is the diffusivity, $L$ is the characteristic length and $u$ is the characteristic velocity.

The other variable required in that equation is the characteristic length which should ideally be the length of the fluid element getting regenerated at the plume surface. Because of the stochastic nature of the process, it is difficult to come up with an accurate value for this length. This is also because this length would depend on the flow conditions, the thickness of the slag layer, the flow rate of the purging gas and possibly 
be a function of the vessel geometry. As a fair estimate, the characteristic length can be taken as some fraction of the spout eye radius.

In a water model study of a gas-injected ladle, Taniguchi et al. [11] performed fluid flow simulations to study the gas-liquid mass transfer in a turbulent flow. The mass transfer coefficient was evaluated using the surface renewal model in which case the characteristic length was taken as the radius of the vessel. That study was for a single liquid phase, hence, any fluid element generated on the surface would travel to the vessel periphery. Taking the characteristic length as the vessel radius was therefore justified in their case. In the present case, however, the presence of a top slag layer would restrict any such movement of the fluid element beyond the spout region. Therefore, a length of half the spout eye radius was chosen as the characteristic length for mass transfer calculations. Using the computed values of the characteristic length and the renewal velocities calculated using CFX, the mass transfer coefficient values were obtained in the range of $1.1 \times 10^{-2} \mathrm{~cm} \mathrm{~s}^{-1}$ to $1.9 \times 10^{-2} \mathrm{~cm} \mathrm{~s}^{-1}$. These values may seem low; a possible explanation is the choice of the characteristic length as half the spout radius. In reality, the eddy length could be much smaller than half the spout radius thereby resulting in higher mass transfer coefficient values.

With known values of the reaction area and the liquid phase mass transfer coefficient, nitrogen absorption was calculated using the mixed control model. To make these computations, a $\mathrm{C}$ language computer program was written and compiled to obtain the increase in nitrogen content of the liquid bulk for different operating conditions. From these calculations, effect of different oxygen and sulfur concentrations and lengths of stirring time were investigated. The starting nitrogen content in the liquid bulk was assumed to be $10 \mathrm{ppm}$. The extent of pickup also depends on the thickness of the slag layer present on the liquid metal, hence different thicknesses of $3 \mathrm{~cm}, 5 \mathrm{~cm}, 7 \mathrm{~cm}$ and 9 $\mathrm{cm}$ were investigated in calculations. Heat transfer effects were ignored in these calculations and the liquid metal was assumed to be at $1873 \mathrm{~K}$. 


\section{Effect of the Oxygen Content}

Due to its surface active nature, presence of oxygen retards the pickup of nitrogen. Figure 27 depict the nitrogen pickup as a function of the gas flow rate for different oxygen concentrations at the spout eye. The liquid metal was assumed to have an initial nitrogen content of $10 \mathrm{ppm}$ and a sulfur content of $0.005 \%$. The stirring was assumed to continue for 20 minutes. It is clear that as the oxygen activity increases, the extent of nitrogen pickup decreases. It is also found that an increasing pickup is possible with a decrease in the slag layer thickness.

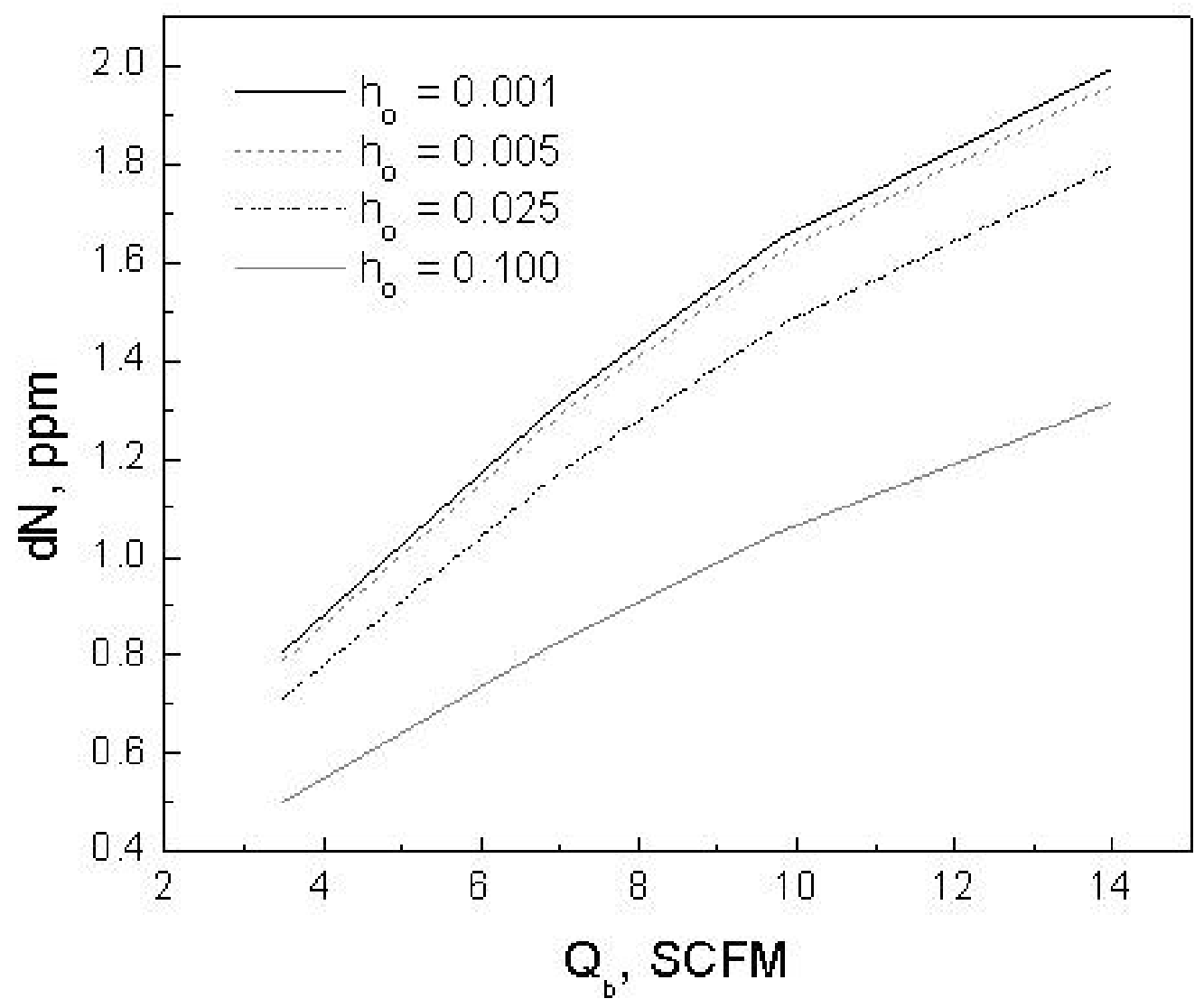

Figure 27, Nitrogen pickup at the spout eye for different oxygen activities, $N_{i}=10 \mathrm{ppm}$, $\% S=0.005$, stirring time $=20$ minutes and slag thickness $=5 \mathrm{~cm}$ 


\section{Effect of Sulfur Content}

Sulfur also acts a surface active element and is responsible for retarding rate of nitrogen dissociation reaction. Nitrogen pickup was calculated as a function of the gas flow rate for different sulfur contents in the metal and is shown in Figure 28. Increasing the sulfur concentration was found to decrease the extent of nitrogen pickup. Same was the case when the slag layer thickness was increased. A total stirring time of 20 minutes was assumed; the oxygen activity was taken as 0.001 . The starting nitrogen content was similarly assumed to be $10 \mathrm{ppm}$.

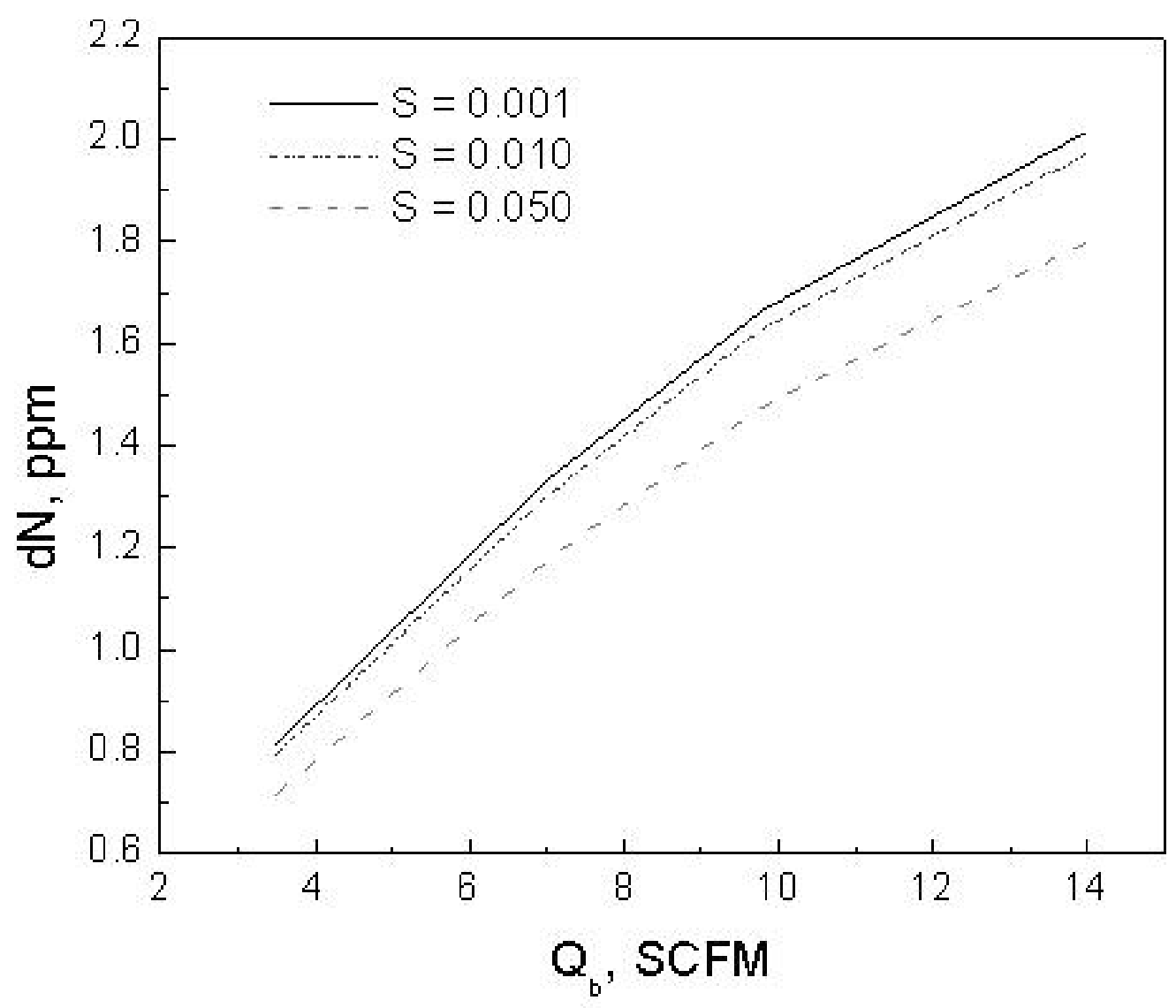

Figure 28, Nitrogen pickup at the spout eye for different sulfur concentrations, $N=10$ ppm, $\mathrm{h}_{\mathrm{O}}=0.001$, stirring time $=20$ minutes and slag thickness $=5 \mathrm{~cm}$ 


\section{Effect of Stirring Time}

Another important parameter which governs the extent of nitrogen pickup from the spout eye is the length of stirring time. It is intuitive that the longer the liquid metal is exposed through the spout eye, the higher will be the gas absorption. The effect of three different stirring intervals of 10,15 and 20 minutes was investigated. It is found from Figure 29 that the nitrogen absorption increases with an increase in the stirring time. It can also be seen that increasing the gas flow rate and decreasing the slag layer thickness also contribute to an increasing nitrogen pickup.

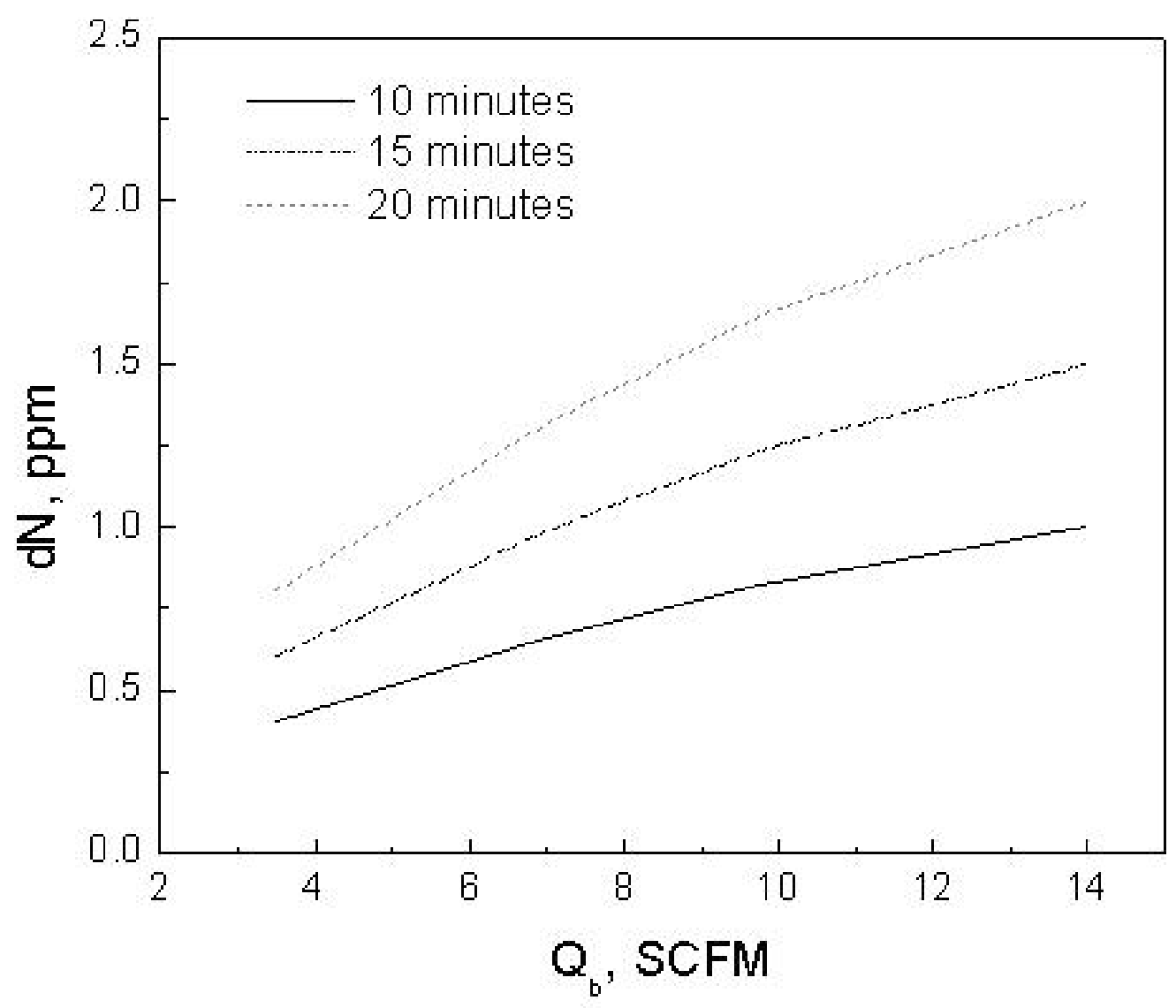

Figure 29, Nitrogen pickup at the spout eye for different stirring intervals, $N=10 \mathrm{ppm}, \%$ $\mathrm{S}=0.005, \mathrm{~h}_{\mathrm{O}}=0.001$ and slag thickness $=5 \mathrm{~cm}$ 


\section{Task V Model for Ladle and Tapping Operations}

The ultimate objective of this research was to come up with a comprehensive model which can predict the pickup of dissolved gases, such as hydrogen and nitrogen, from their various sources. In order to formulate this all-inclusive model, results have been incorporated from the experimental findings and the various sub-models developed in this research into a number of correlative equations. Needless to say, these equations are empirical rather than fundamental in nature and they may be valid in a limited range of application due to obvious limitations. Nonetheless, they are derived from fundamental principles discussed in this research and therefore try to accommodate various parameters and variable which may affect the pickup of these gases.

\section{Hydrogen Pickup}

The different sources of hydrogen investigated in this research include

1. Hydrated $\mathrm{CaO}\left(\mathrm{Ca}(\mathrm{OH})_{2}\right)$ addition during ladle operations

2. Carburizer addition

3. Atmospheric moisture during tapping of liquid steel

It was found that close to $2 \%$ of the hydrogen added in the form of $\mathrm{Ca}(\mathrm{OH})_{2}$ went into the liquid steel. Maximum pickup scenario can be when all the lime added during steelmaking is hydrated. In such a case, the amount of hydrogen picked up is given by

$$
\Delta H=\frac{2 * 2 * W t \cdot \lim e}{74 * W} * 10^{4}, \text { ppm } \quad \text { Equation } 21
$$

where Wt.lime is the weight of lime $(\mathrm{kg}), \mathrm{W}$ is the weight of liquid metal $(\mathrm{kg})$, molecular weight of $\mathrm{Ca}(\mathrm{OH})_{2}$ is 74 and that of $\mathrm{H}_{2}$ is $2 \mathrm{~kg}$ per kilo mole.

A recovery of 8 to $12 \%$ was observed in the experiments on hydrogen pickup from petroleum coke. It is therefore reasonable to say that close to $10 \%$ of the hydrogen present in the form of impurity in the coke results into liquid steel. Hence, the amount of hydrogen pickup from coke additions is given as 


$$
\Delta H=\frac{10 * x * W t . \text { Coke }}{100 * W} * 10^{4}, \text { ppm } \quad \text { Equation } 22
$$

where Wt.coke is the weight coke $(\mathrm{kg}), \mathrm{W}$ is the weight of liquid metal $(\mathrm{kg})$ and $\mathrm{x}$ is the percentage impurity of hydrogen in the coke sample.

The hydrogen picked up during the tapping of liquid steel depends on the oxygen and sulfur content of the liquid metal along with the partial pressure of water vapor in the atmosphere. In order to derive an empirical correlation, hydrogen pickup was calculated for different values of oxygen (0.001 to $0.1 \mathrm{wt} \%)$, sulfur (0.001 to $0.1 \mathrm{wt} \%$ ) and water vapor pressure (0.002 to 0.04 atmospheres). Regression analysis was subsequently used and the most suitable correlation obtained which is given below

$$
\begin{array}{rlr}
\ln (\Delta H & =0.6225-0.4647 \cdot \ln \left(h_{O}\right)-0.2037 \cdot \ln (S)+0.6687 \cdot \ln \left(p_{\mathrm{H}_{2} \mathrm{O}}\right) \\
& +0.0589 \cdot\left[\ln \left(h_{O}\right)\right]^{2}-0.0291 \cdot[\ln (S)]^{2}-0.0076 \cdot\left[\ln \left(p_{\mathrm{H}_{2} \mathrm{O}}\right)\right]^{2} & \text { Equation } 23 \\
& +0.005 \cdot\left[\ln \left(h_{O}\right)\right]^{3}-0.00142 \cdot[\ln (S)]^{3}+0.000085 \cdot\left[\ln \left(p_{\mathrm{H}_{2} \mathrm{O}}\right)\right]^{3} &
\end{array}
$$

where $h_{0}$ is the oxygen content (wt\%), $S$ is the sulfur content (wt\%), $p_{\mathrm{H}_{2} \mathrm{O}}$ is the partial pressure of $\mathrm{H}_{2} \mathrm{O}$ in the atmosphere and $\Delta \mathrm{H}$ is the hydrogen pickup in ppm.

\section{Nitrogen Pickup}

The different sources of nitrogen investigated in this research are as follows
1. Ferroalloys
2. Carburizers
3. Transport through the slag layer
4. Pickup during tapping
5. Pickup from the spout eye

Experiments investigating nitrogen pickup from ferroalloy additions demonstrated that all of the nitrogen present as an impurity entered the liquid metal. The average 
nitrogen pickup from pitch coke was around $20 \%$. The nitrogen pickup from steelmaking additions can therefore be represented as

$$
\Delta N=\left(\frac{20 * x * W t \cdot \text { Coke }}{100 * W}+\frac{y * W t . \text { Alloy }}{W}\right) * 10^{4}, \text { ppm } \quad \text { Equation } 24
$$

where Wt.Coke is the weight of coke $(\mathrm{kg})$, Wt.Alloy is the weight of ferroalloy addition $(\mathrm{kg}), \mathrm{W}$ is the weight of liquid metal $(\mathrm{kg}), \mathrm{x}$ is the percentage impurity of nitrogen in the coke sample and $y$ is the percentage impurity of nitrogen in the ferroalloy.

Nitrogen pickup from the atmosphere was investigated from three sources; transfer through the slag layer, pickup during tapping and pickup from the spout eye. Nitrogen pickup due to its transfer through the slag layer was found to be very slow. Only a few ppm of nitrogen was absorbed over a time span of 20 hours. Such a time interval is unrealistic in steelmaking operations hence the nitrogen pickup through such a mechanism can be neglected.

During the tapping of liquid steel, the extent of nitrogen picked up from entrained air bubbles depends on the oxygen and sulfur content of the liquid metal. It is intuitive that the pickup would depend on the tapping rate (or the inlet velocity into the ladle). In order to investigate the effect of different tapping rates, CFD simulations were run to compare the total bubble area for different inlet velocities. It was found that decreasing the liquid velocity proportionately decreased the total bubble area. In other words, a change in the tapping rate was compensated by an equal change in the reaction area. The total nitrogen pickup was therefore not a function of the tapping rate. The tapping rates investigated were between 1 tonne/sec and 0.5 tonne/sec, which represent typical tapping rates for tapping of liquid steel. Equation (25) represents the nitrogen pickup predicted for a range of oxygen ( 0.001 to $0.1 \mathrm{wt} \%$ ) and sulfur (0.001 to $0.1 \mathrm{wt} \%$ ) content in the liquid metal.

$$
\begin{aligned}
\Delta N= & 20.252-107.979 \cdot\left(h_{o}\right)-49.651 \cdot(S) \quad \text { Equation } 25 \\
& +298.849 \cdot\left(h_{o}\right)^{2}+77.06 \cdot(S)^{2} \quad
\end{aligned}
$$


where $h_{b}$ is the oxygen content (wt\%), $\mathrm{S}$ is the sulfur content (wt\%) and $\Delta \mathrm{N}$ is the nitrogen pickup in ppm.

Nitrogen absorption from the spout eye was also estimated for different operating parameters. The variables which affect the extent of pickup include the oxygen and sulfur content of the metal, the inert gas flow rate, the thickness of the slag layer and the total stirring time. A range of values was used for these variables to represent a typical stirring operation and a correlation was obtained using a multiple linear regression analysis and is given as follows.

$$
\begin{aligned}
\ln (\Delta N)= & -3.936-0.4019 \cdot \ln \left(h_{O}\right)-0.212 \cdot \ln (S)+0.944 \cdot \ln \left(Q_{b}\right) \\
& -1.111 \cdot \ln (\text { slagtk })+1.002 \cdot \ln (t)-0.0341 \cdot\left[\ln \left(h_{O}\right)\right]^{2} \\
& -0.018[\ln (S)]^{2}-0.064\left[\ln \left(Q_{b}\right)\right]^{2}-0.1385 \cdot[\ln (\text { slagtk })]^{2} \quad \text { Equation } 26 \\
& -0.00048 \cdot[\ln (t)]^{2}
\end{aligned}
$$

where $h_{0}$ is the oxygen content investigated in the range of $(0.001$ to $0.1 \mathrm{wt} \%), \mathrm{S}$ is the sulfur content (0.001 to $0.1 \mathrm{wt} \%), \mathrm{Q}_{\mathrm{b}}$ is the gas flow rate (3.5 to $\left.14 \mathrm{NLPM}\right)$, slagtk is the slag layer thickness ( 3 to $9 \mathrm{~cm}$ ) and t is the stirring time (10 to 20 minutes).

As an example, consider 200 tonnes of liquid steel is being tapped at 0.5 tonnes per second with oxygen and sulfur contents of 0.008 and $0.005 \mathrm{wt} \%$ respectively. The temperature and humidity levels for the day set the partial pressure of water vapor to 0.02 atmospheres. At the ladle metallurgy station, around $200 \mathrm{~kg}$ of hydrated lime and $100 \mathrm{~kg}$ of petroleum coke, with $0.3 \%$ hydrogen impurity and $1.0 \%$ nitrogen impurity, are added. Also added is $50 \mathrm{~kg}$ of medium carbon ferromanganese with $0.1 \%$ nitrogen impurity. The liquid metal is continuously stirred at an argon flow rate of 10 SCFM for the 15 minutes the ladle is at the station. To prevent gas-metal reaction a $5 \mathrm{~cm}$ slag layer is present on the liquid metal. Using the previous empirical equations, the total hydrogen and nitrogen pickup in these situations is calculated to be 4.8 and $21.66 \mathrm{ppm}$ respectively. Considering a different case, let the steel not be deoxidized and desulfurized before tapping and the oxygen and sulfur levels are 0.05 and $0.02 \mathrm{wt} \%$ respectively. Some more slag is added to increase the slag layer thickness to $7 \mathrm{~cm}$ and 
the stirring is continued for 2 more minutes. The humidity level increases and the water vapor pressure is now 0.03 atmospheres. In such a case the total hydrogen and nitrogen pickup values will possibly be 2.2 and $16.49 \mathrm{ppm}$.

It is therefore demonstrated that the empirical correlations can be used to calculate the total hydrogen and nitrogen pickup from the different steelmaking additions as well as from the atmosphere during the tapping and ladle operations. 


\section{Bibliography}

1. Fruehan, R. J., "Making Shaping and Treating of Steel", $11^{\text {th }}$ Edition, AISE Steel Foundation, Pittsburgh, USA, 1998.

2. Gaskell, D. R., "Introduction to the Thermodynamics of Materials", $3^{\text {rd }}$ Edition, Taylor \& Francis, 1995, pp. 389

3. B. Deo. and R. Boom., "Fundamentals of Steelmaking Metallurgy", Prentice Hall International, London, U.K., 1993.

4. Ozturk, B. and Fruehan, R. J., "Reaction of Nitrogen between Calcium Aluminate Slags and Fe-Al alloys", Ironmaking and Steelmaking, Vol. 15, No. 6, 1988, pp. 305-310

5. Eisenhuttenleute, V. D., "Slag Atlas", $2^{\text {nd }}$ Edition, Verlag Stahleisen GbmH, 1995

6. Turkdogan, E. T., "Fundamentals of Steelmaking", The Institute of Metals, 1996

7. Fruehan, R. J. and Martonik, L. J., "The Rate of Absorption of Hydrogen into Iron and of Nitrogen into Fe-Cr and Fe-Ni-Cr Alloys Containing Sulfur", Metallurgical Transactions Vol. 12B, 1981 pp. 379-384

8. William T Lankford, Jr. et. al., "The Making, Shaping and Treating of Steel", $10^{\text {th }}$ Edition, USS and AISE, 1985

9. Goldstein, D., "Nitrogen Reactions in the EAF and OSM", Ph. D. Thesis, 1996

10. Subagyo, Brooks, G. A. and Irons, G. A., "Spout Eye Area Correlation in Ladle Metallurgy", ISIJ International Vol. 43, No. 2, 2003, pp. 262-263

11. Taniguchi, S., Matsukura, Y. and Kikuchi, A., "Turbulent Flow and Gas-Liquid Mass Transfer in Gas-injected System", Tetsu-to-Hagane, Vol. 86, no. 4, 2000, pp. 210-216 